\title{
Energy Levels and Transition Probabilities in Doubly-lonized Erbium (Er III)
}

\author{
Jean-François Wyart, ${ }^{1}$ Jean Blaise, ${ }^{1}$ William P. Bidelman ${ }^{2}$ and Charles R. Cowley ${ }^{3}$ \\ ${ }^{1}$ Laboratoire Aimé Cotton," Bât. 505, CNRS II, Centre universitaire, F-91405, Orsay, France \\ 2 Department of Astronomy, Case Western Reserve University, Cleveland OH 44106-7215, U.S.A. \\ 3 Department of Astronomy, University of Michigan, Ann Arbor, MI 48109-1090, U.S.A.
}

Received January 15, 1997; accepted in revised form March 19, 1997

PACS Ref: $3220 \mathrm{~J}, 9730$

\begin{abstract}
The spectrum of Er III reported by Becher (1966) was reanalysed with the support of new predictions of energies and transition probabilities. The number of energy levels was increased from 45 to 115 , including two levels of $4 f^{11} 7 \mathrm{~s}$ and the levels ${ }^{3} \mathrm{~F}_{3},{ }^{3} \mathrm{~F}_{2}$ and ${ }^{1} \mathrm{G}_{4}$ of the ground configuration $4 \mathrm{f}^{12}$. All 470 classified lines are reported with transition probabilities for most of them. Several of these lines had not yet been attributed to Er III in the spectrum of the star HR 465 .
\end{abstract}

\section{Introduction}

The initial steps in the analysis of doubly-ionized erbium have been summarized in 1978 in the critical compilation by Martin, Zalubas and Hagan [1] and are briefly recalled here. The main features of Er III had been published by Spector, who reported 137 lines classified by 45 levels including the lowest ones of $4 \mathrm{f}^{12}, 4 \mathrm{f}^{11} 6 \mathrm{p}, 4 \mathrm{f}^{11} 6 \mathrm{~s}$ and $4 \mathrm{f}^{11} 5 \mathrm{~d}$ [2]. A few corrections and additions resulting from parametric studies of $4 f^{11}(5 d+6 s)$ [3] and of $4 f^{11} 6 p$ [4] were taken into account in [1]. After 1978, the even levels of $\mathrm{Er}$ III were used in a global interpretation of the $4 f^{N}(5 d+6 s)$ configurations, but no extension was attempted in the classification [5]. The need for new spectroscopic data in the spectral interpretation of chemically peculiar stars is an incentive for resuming studies in doubly-ionized lanthanides. The first analysis of Dy III was achieved recently [6] and a brief summary of related problems was given in [7]. Moreover erbium is already known to contribute to more than 300 lines in the spectrum of the chemically peculiar star HR 465 [8].

The only available data for extending the analysis of $\mathrm{Er}$ III are in the dissertation by Becher [9] which had been kindly put at our disposal by H. M. Crosswhite when Er I and II were analysed [10]. The included linelist comprises about 3800 wavelengths from 2036 to $8725 \AA$ which are reported with intensities in three excitations (microwave discharge, d.c. arc and mild spark). We had noticed already that, in spite of these intensity comparisons, the ionization assignments of [9] were partly erroneous in the long wavelength region. Nevertheless the wavenumber accuracy was good enough for energy level searching as it has been proved by Becher himself who first classified some of the

* The laboratoire Aimé Cotton is in association with Université Paris-Sud. strongest $6 s-6 p$ transitions and by van Kleef who found the ${ }^{3} \mathrm{H}_{4}$ level of $4 \mathrm{f}^{12}$.

\section{New energy levels}

We used the computer codes of Bordarier, Bachelier and Sinzelle [11] and those by Cowan [12] for calculating the energy levels in the Slater-Condon approach and for deriving transition probabilities from the eigenvectors in intermediate coupling. The transition probabilities were used as a guide for finding new levels. They compare fairly well with the observed intensities if the comparison runs on a limited range of wavelengths due to at least two facts. Intensity estimates are generally not corrected from plate sensitivity factors and Becher's observations span from ultraviolet to near infrared. The transition probabilities agree better with the intensity figures of Spector, but the linelist of [2] reports classified lines only which limits the comparisons.

The coupling conditions $\left[\left(4 \mathrm{f}^{N} \alpha S_{1}, L_{1}\right) J_{1}, n l j_{2}\right] J$ are wellobeyed in $4 \mathrm{f}^{11} 6 \mathrm{~s}$ and $4 \mathrm{f}^{11} 6 \mathrm{p}$ and, due to selection rules, the levels cannot be found from many transitions. This is not true for $4 \mathrm{f}^{11} 5 \mathrm{~d}$ which is far from $L S$ and from $\left(\alpha J_{1} j_{2}\right)$ conditions, according to the results of the present theoretical studies.

Only four levels of the ground configuration $4 \mathrm{f}^{12}$ had been reported earlier. Three new levels, ${ }^{3} \mathrm{~F}_{3},{ }^{3} \mathrm{~F}_{2}$ and ${ }^{1} \mathrm{G}_{4}$, were found from their strongest predicted transitions with $4 \mathrm{f}^{11} 5 \mathrm{~d}$ and they classify now 24 lines in Table I. The fine structure of ${ }^{3} \mathrm{H}$ and ${ }^{3} \mathrm{~F}$ intervals are only slightly larger in Er III than they are in $4 f^{12} 6 s^{2}$ of Er I, which is a usual situation for lanthanides. A total of seven levels made it possible to derive the energy parameters of $4 f^{12}$ with limited constraints. All effective parameters $\alpha, \beta, \gamma$ of the linear correction for far configuration interaction $\alpha L \cdot(L+1)+10 \beta G(R 7)+12 \gamma G(G 2)$ were fixed as well as the ratio of electrostatic parameters $E_{1} / E_{3}$. This led to predictions for the 6 missing levels. The lowest of them $\left({ }^{1} \mathrm{D}_{2}\right)$ is expected near $24900 \mathrm{~cm}^{-1}$ and its determination should not be possible without further observations of the spectrum.

The upper even configuration $4 f^{11} 6 \mathrm{p}$ is accurately interpreted with the same Hamiltonian as for other $4 f^{N} 6 p$ configurations of doubly-ionized lanthanides, configuration mixing effects being taken into account by $\alpha, \beta, \gamma$, and by the correction $\alpha_{\text {TOT }} L \cdot(L+1)$ which is equivalent to a Slater- 
type parameter $F^{1}(f, p)$ [13]. The r.m.s. deviation for 35 levels $\left(21 \mathrm{~cm}^{-1}\right)$ is worse than it was in the first study which involved 18 levels built on $4 f^{114} I_{15 / 2,13 / 2,11 / 2}$ only. Nevertheless it is established now that the predicted levels of [4] were about $80 \mathrm{~cm}^{-1}$ too low for $4 \mathrm{f}^{11}\left({ }^{4} \mathrm{I}\right) 6 \mathrm{p}$ and $250 \mathrm{~cm}^{-1}$ too low for $4 f^{11}\left({ }^{4} F\right) 6 p$, which illustrates the difficulties of parametric studies performed with few levels. It is remarkable that all the known levels have $\left(J_{1}, j_{2}\right)$ first components larger than $50 \%$ and that all $\left(J_{1}, j_{2}\right)$ multiplets given in Table II are separated in energy.

In the odd parity both the flatness of some $J_{-j}$ multiplets and the overlap of $4 \mathrm{f}^{11} 5 \mathrm{~d}$ and $4 \mathrm{f}^{11} 6 \mathrm{~s}$ made some difficulties in the identifications, "forbidden" $4 \mathrm{f}-6 \mathrm{~s}$ transitions being obviously present. A few undeterminacies of $J$-values were removed with the support of the computed transition probabilities, the calculation of which progressed in parallel with the classification. It is seen from the total percentages of $4 \mathrm{f}^{11} 6 \mathrm{~s}$ components in the eigenfunctions of levels given in Table II, that at least four couples of $4 \mathrm{f}^{11} 6 \mathrm{~s}$ and $4 \mathrm{f}^{11} 5 \mathrm{~d}$ levels mix significantly. This explains the well-defined value of the interaction integral $R^{3}(4 \mathrm{f} 5 \mathrm{~d}, 6 \mathrm{~s} 4 \mathrm{f}): 2879 \pm 297 \mathrm{~cm}^{-1}$ reported in Table III with other parameters, the direct integral $R^{2}(4 \mathrm{f} 5 \mathrm{~d}, 4 \mathrm{f} 6 \mathrm{~s})$ being loosely defined and eliminated. From the coefficients of the $R^{3}$ parameter in intermediate coupling, the shifts pertaining to this interaction were evaluated. They nowhere exceed $215 \mathrm{~cm}^{-1}$ which is large with regard to the r.m.s. deviation of the present study $\left(35 \mathrm{~cm}^{-1}\right)$ but keeps $4 f 5 \mathrm{~d}-4 \mathrm{f} 6 \mathrm{~s}$ a small interaction in comparison with others in the lanthanides (for example $5 \mathrm{~d}^{2}-5 \mathrm{~d} 6 \mathrm{~s}$ or $5 \mathrm{~d} 6 \mathrm{p}$ 6s6p). In Table III, the parameters which describe far configuration mixing effects on the terms of $l^{N} l^{\prime}\left(D^{3}, Y^{2}, Y^{4}\right)$ are the direct and exchange "forbidden Slater parameters" giving according to the definitions of [12] and $\alpha_{\text {TOT }}$ is equivalent to $D^{1}$.

Transitions with upper levels above $79000 \mathrm{~cm}^{-1}$ classify weak lines and the correspondence between levels and theoretical energies become ambiguous. For this reason, the odd levels reported in Table II in the range $41000-50000 \mathrm{~cm}^{-1}$ are attributed qualitative designations only. A successful attempt was made to locate the transition array $4 \mathrm{f}^{11}\left({ }^{4} \mathrm{I}_{15 / 2}\right) 7 \mathrm{~s}-4 \mathrm{f}^{11}\left({ }^{4} \mathrm{I}_{15 / 2}\right) 6 \mathrm{p}$ and the intensity pattern of the seven classified $6 \mathrm{p}-7 \mathrm{~s}$ lines fit very well to the theoretical $g \mathrm{~A}$ values. Owing to the very regular trends of the $6 \mathrm{~s}-7 \mathrm{~s}$ electron jump along the lanthanide period already used in [14], there is no need revising the ionization energy predicted by Sugar and Reader.

\section{Classified lines and transition probabilities}

The classified lines in Table I are reported with transition probabilities $g A$ calculated by means of [12] in three separate studies of the arrays $4 \mathrm{f}^{11} 6 \mathrm{p}-4 \mathrm{f}^{11} 7 \mathrm{~s}, 4 \mathrm{f}^{12}$ $4 f^{11} 5 d+4 f^{11} 6 s, 4 f^{11} 6 p-4 f^{11} 5 d+4 f^{11} 6 s$. Cut-off values of $g A$ were chosen to keep about the 1500 strongest lines in the latter array. It explains that some $g A$ values given for $4 \mathrm{f}-5 \mathrm{~d}$ transitions are smaller than the discarded $g A$ values of 6s-6p transitions, the radial dipole transition integrals being (in a.u.) $(4 \mathrm{f}|r| 5 \mathrm{~d})=1.123, \quad(6 \mathrm{p}|r| 6 \mathrm{~s})=-3.271 \quad$ and $(6 \mathrm{p}|r| 5 \mathrm{~d})=-2.776$.

The 482 reported transitions correspond to 470 lines, but for several of the 12 doubly-classified lines, the $g A$ value should indicate the dominant transition. The average devi- ation between the measured wavelengths and those derived from the energy levels is $0.004 \AA$. Some large discrepancies occur for very strong transitions to the ground configuration.

\section{Er III in stellar spectra}

The third spectrum of erbium is known to be present in the spectra of chemically peculiar stars of the upper main sequence. Aikman, Cowley and Crosswhite [16] find Er III by coincidence statistics in four of five stars with third spectra of the lanthanides. It is probably present in the fifth star as well. HR 465 at the time of its rare earth maximum is particularly well suited for line identification work because of the sharpness of the spectral lines. Moreover, Bidelman's [8] plates have unusually good coverage in the near ultraviolet.

The present analysis has resulted in some thirty modifications of the published identification list for Er III. These changes range from an indication that a previously observed laboratory line is now classified, to entirely new identifications of stellar features. The changes will be incorporated in the electronic list a vailable from the University of Michigan (CRC's home page). They are listed in Table IV.

An open question is described below. The transition between the ground level $4 \mathrm{f}^{12}{ }^{3} \mathrm{H}_{6}$ and the third level with $J=6$ in $4 \mathrm{f}^{11} 5 \mathrm{~d}$, named $\left({ }^{4} \mathrm{I}_{13 / 2}, \mathrm{~d}_{3 / 2}\right)$ is predicted at $3638.977 \AA$ It is lacking in both laboratory linelists $[2,9]$ although its probability is high. The corresponding line in the $4 \mathrm{f}^{12} 6 \mathrm{~s}^{2}-4 \mathrm{f}^{11} 5 \mathrm{~d} 5 \mathrm{~s}^{2}$ transition array of Er I, where the $6 \mathrm{~s}^{2}$ closed shell does not change the coupling conditions for the lowest multiplets, occurs at $6221 \AA$ It is the strongest Er I line observed above $5827 \AA$ according to [15]. In the spectrum of HR 465 [8], an intense $(I=5 \mathrm{~d}$ ?) unassigned line at $3639.01 \AA$ fits the Er III expected wavelength. For clarifying this point, and for resuming the search for Er III levels on better grounds, new laboratory observations are needed.

\section{Acknowledgements}

The authors are indebted to $\mathrm{H}$. M. and $\mathrm{H}$. Crosswhite who kindly provided them with the linelists needed in this study. The first authors are grateful to A. Bachelier and J. Sinzelle who enable them to perform the parametric calculations of Er III at the C.R.I. computer centre of Paris-Sud university. The revision of lanthanide spectra for plasma diagnostic purposes is part of a project supported by the Scientific and Environmental Affairs Division of the North Atlantic Treaty Organization (Grant CRG930793 acknowledged by the first authors.

\section{References}

1. Martin, W. C., Zalubas, R. and Hagan, L., "Atomic Energy Levels The Rare-Earth Elements", NSRDS-NBS 60 (1978).

2. Spector, N., J. Opt. Soc. Am. 63, 358 (1973).

3. Wyart, J.-F., Blaise, J. and Camus, P., Physica Scripta 9, 325 (1974).

4. Wyart, J.-F., Koot, J. J. A. and van Kleef, Th. A. M., Physica C77, 159 (1974).

5. Wyart, J.-F. and Bauche-Arnoult, C., Physica Scripta 22, 583 (1981).

6. Spector, N., Sugar, J. and Wyart, J.-F., J. Opt. Soc. Am. B14, 511 (1997).

7. Wyart, J.-F, in Proceedings of "Laboratory and Astronomical High Resolution Spectra", (Edited by A. J. Sauval, R. Blomme and N. Grevesse) A.S.P.C. Conf. Series, vol. 81, pp. 182-95 (1995). 
8. Bidelman, W. P., Cowley, C. R. and Iler, A. L., "Wavelength Identification in the Magnetic C.P. Star HR $465^{\prime \prime}$, Publ. Obs. Univ. Mich., vol. XII No. 3 (1995).

9. Becher, J., Thesis, Johns Hopkins Univ., Baltimore 134pp (1966).

10. van Kleef, Th. A. M., Koot, J. J. A. and Wyart, J.-F., unpublished analysis (1975) quoted in [1].

11. Bordarier, Y., Bachelier, A. and Sinzelle, J., Chain of Programs AGENAC, ASSAC, DIAGAC and GRAMAC, unpublished, Orsay (1980).
12. Cowan, R. D., "The Theory of Atomic Structure and Spectra" (Univ. of California Press, Berkeley 1981) and computer codes.

13. Wyart, J.-F., J. Opt. Soc. Am. 68, 197 (1978).

14. Sugar, J. and Reader, J., J. Chem. Phys. 59, 2083 (1973).

15. Meggers, W. F., Corliss, C. H. and Scribner, B. F., "Tables of Spectral Lines Intensities", NBS Monograph 145 (1975).

16. Aikman, G. C. L., Cowley, C. R. and Crosswhite, H. M., Astrophys. J. 232, 812 (1979) 
Table I. Classified lines of Er III. The successive columns are: (1) the air wavelength $\lambda_{\exp }$ (in $\AA$ ) from [9] unless indicated, (2) the intensity, (3) the vacuum wavenumber (in $\mathrm{cm}^{-1}$ ), (4) the difference $\lambda_{\exp }-\lambda_{\mathrm{RITZ}}, \lambda_{\mathrm{RITZ}}$ being calculated from the levels, (5) the even energy level $E^{\mathrm{e}}$, (6) the quantum number $J^{\mathrm{e}}$, (7) the odd energy level $E^{0}$ and (8) the quantum number $J^{0}$, (9) the labels of both levels as given in Table II (a condensed physical designation), (10) the transition probability $g A$ (in $\left.\mathrm{s}^{-1}\right), g$ being the statistical weight of the upper level; an eventual note is explicited at the end of the Table.

\begin{tabular}{|c|c|c|c|c|c|c|c|c|}
\hline (1) & (2) & (3) & (4) & (5) & (6) (7) & (8) & (9) & (10) \\
\hline 6824.295 & 80 & 14649.49 & 0.009 & 18383.59 & 4-33033.10 & 4 & $1 G-d$ & \\
\hline 6644.005 & 80 & 15047.01 & -0.004 & 6969.80 & $5-22016.80$ & 5 & $3 \mathrm{H}-\mathrm{I} 7 \mathrm{~d} 5$ & $2.279(5)$ \\
\hline 6488.670 & 48 & 15407.22 & -0.013 & 10785.51 & $4-26192.70$ & 5 & $3 \mathrm{H}-\mathrm{I} 6 \mathrm{~d} 3$ & $1.041(6)$ \\
\hline 6393.635 & 80 & 15636.23 & 0.037 & 6969.80 & $5-22606.12$ & 6 & $3 \mathrm{H}-17 \mathrm{~d} 5$ & $2.618(5)$ \\
\hline 6302.225 & 41 & 15863.02 & 0.024 & 10785.51 & $4-26648.59$ & 4 & $3 \mathrm{H}-15 \mathrm{~d} 3$ & \\
\hline 6153.460 & 48 & 16246.52 & 0.037 & 13219.80 & $2-29466.42$ & 3 & $3 F-d$ & \\
\hline 5988.439 & 70 & 16694.22 & 0.021 & 55547.30 & $7-38853.02$ & 6 & I7p1- & \\
\hline 5903.279 & 80 & 16935.04 & -0.004 & 5081.77 & $4-22016.80$ & 5 & $3 F-17 d 5$ & $1.464(6)$ \\
\hline 5881.820 & 80 & 16996.83 & 0.007 & 12472.55 & $3-29469.40$ & 4 & 3F-Id & $1.075(6)$ \\
\hline 5851.362 & 46 & 17085.30 & 0.007 & 10785.51 & $4-27870.83$ & 5 & $3 \mathrm{H}-16 \mathrm{~d} 5$ & $3.462(5)$ \\
\hline 5621.564 & 70 & 17783.71 & 0.000 & 18383.59 & $4-36167.30$ & 3 & $1 G-d$ & \\
\hline 5570.362 & 80 & 17947.17 & 0.016 & 18383.59 & $4-36330.81$ & 5 & $1 G-d$ & $3.519(5)$ \\
\hline 5471.313 & 48 & 18272.07 & -0.018 & 18383.59 & $4-36655.60$ & 5 & $1 G-d$ & \\
\hline 5469.627 & 80 & 18277.70 & -0.009 & 12472.55 & $3-30750.22$ & 4 & $3 F-d$ & 2 \\
\hline 5350.720 & 90 & 18683.88 & 0.003 & 10785.51 & $4-29469.40$ & 4 & 3H-Id & \\
\hline 5286.634 & 42 & 18910.37 & 0.018 & 55547.30 & $7-36636.87$ & 7 & I7p1-F4d5 & \\
\hline 5255.920 & 20 & 19020.87 & 0.028 & 10785.51 & $4-29806.48$ & 5 & $3 \mathrm{H}-\mathrm{I} 5 \mathrm{~s} 1$ & 2 \\
\hline 5204.147 & 70 & 19210.09 & 0.005 & 10785.51 & $4-29995.62$ & 5 & $3 \mathrm{H}-\mathrm{I} 5 \mathrm{~d} 3$ & \\
\hline 5200.680 & 80 & 19222.90 & 0.000 & 6969.80 & $5-26192.70$ & 5 & $3 \mathrm{H}-16 \mathrm{~d} 3$ & \\
\hline 5200.236 & 90 & 19224.54 & -0.003 & 18383.59 & $4-37608.12$ & 4 & $1 G-d$ & $6.019(5)$ \\
\hline 5157.864 & 47 & 19382.47 & -0.002 & 65934.64 & $5-46552.18$ & 5 & I5p1-?d & \\
\hline 5145.340 & 80 & 19429.65 & 0.008 & 12472.55 & $3-31902.23$ & 4 & $3 F-I 4 s 1$ & $1.201(6)$ \\
\hline 4876.062 & 59 & 20502.63 & 0.007 & 6969.80 & $5-27472.46$ & 6 & $3 \mathrm{H}-16 \mathrm{~d} 3$ & \\
\hline 4862.333 & 80 & 20560.51 & 0.010 & 12472.55 & $3-33033.10$ & 4 & $3 F-d$ & $1.216(6)$ \\
\hline 4826.538 & 41 & 20713.00 & 0.022 & 12472.55 & $3-33185.64$ & 3 & $3 F-F 4 d 3$ & \\
\hline 4783.122 & 80 & 20901.00 & 0.006 & 6969.80 & $5-27870.83$ & 5 & $3 \mathrm{H}-\mathrm{I} 6 \mathrm{~d} 5$ & $3.732(6)^{\mathrm{a}}$ \\
\hline 4749.491 & 43 & 21049.00 & 0.034 & 67986.38 & $5-46937.23$ & 4 & $16 \mathrm{p} 3-d$ & \\
\hline 4746.858 & 41 & 21060.68 & -0.007 & 10785.51 & $4-31846.16$ & 3 & $3 \mathrm{H}-\mathrm{I} 4 \mathrm{~d} 5$ & \\
\hline 4735.554 & 80 & 21110.95 & -0.005 & 5081.77 & $4-26192.70$ & 5 & $3 F-16 \mathrm{~d} 3$ & $9.393(6)$ \\
\hline 4734.225 & 70 & 21116.74 & -0.004 & 10785.51 & $4-31902.23$ & 4 & $3 \mathrm{H}-\mathrm{I} 4 \mathrm{~s} 1$ & \\
\hline 4694.172 & 41 & 21297.05 & 0.020 & 68234.37 & $4-46937.23$ & 4 & I4p1-d & \\
\hline 4672.716 & 41 & 21394.84 & 0.031 & 68332.21 & $5-46937.23$ & 4 & I4p1-d & \\
\hline 4669.915 & 80 & 21407.67 & 0.002 & 10785.51 & 4-32193.19 & 5 & $3 \mathrm{H}-\mathrm{I} 4 \mathrm{~s} 1$ & $8.742(5)$ \\
\hline 4669.094 & 80 & 21411.44 & 0.002 & 10785.51 & 4-32196.96 & 4 & $3 \mathrm{H}-\mathrm{d}$ & $1.029(6)$ \\
\hline 4612.932 & 80 & 21672.11 & 0.000 & 55547.30 & $7-33875.19$ & 6 & $\mathrm{I} 7 \mathrm{p} 1-\mathrm{I} 5 \mathrm{~d} 5$ & \\
\hline 4589.485 & 47 & 21782.83 & 0.006 & 18383.59 & 4-40166.45 & 4 & $1 \mathrm{G}-\mathrm{F} 3 \mathrm{~s} 1$ & \\
\hline 4584.224 & 70 & 21807.83 & 0.023 & 6969.80 & $5-28777.74$ & 6 & $3 \mathrm{H}-\mathrm{I} 6 \mathrm{~d} 5$ & $8.702(6)$ \\
\hline 4579.808 & 80 & 21828.86 & 0.000 & 10785.51 & 4-32614.37 & 5 & $3 \mathrm{H}-\mathrm{d}$ & $8.357(5)$ \\
\hline 4540.722 & 80 & 22016.76 & 0.008 & 0.00 & $6-22016.80$ & 5 & $3 \mathrm{H}-\mathrm{I} 7 \mathrm{~d} 5$ & $1.181(6)$ \\
\hline 4539.198 & 39 & 22024.15 & -0.004 & 18383.59 & $4-40407.72$ & 3 & $1 G-d$ & \\
\hline 4497.582 & 80 & 22227.93 & 0.006 & 61493.77 & $6-39265.81$ & 5 & $17 \mathrm{p} 3-$ & \\
\hline 4493.610 & 80 & 22247.58 & 0.002 & 10785.51 & $4-33033.10$ & 4 & $3 \mathrm{H}-\mathrm{d}$ & $2.082(6)$ \\
\hline 4471.890 & 80 & 22355.64 & 0.026 & 55547.30 & $7-33191.53$ & 6 & I7p1-d & \\
\hline 4463.014 & 80 & 22400.10 & 0.006 & 10785.51 & $4-33185.64$ & 3 & $3 \mathrm{H}-\mathrm{F} 4 \mathrm{~d} 3$ & \\
\hline 4443.278 & 80 & 22499.59 & 0.002 & 6969.80 & $5-29469.40$ & 4 & $3 \mathrm{H}-\mathrm{Id}$ & $6.334(5)$ \\
\hline 4422.368 & 80 & 22605.97 & 0.029 & 0.00 & $6-22606.12$ & 6 & $3 \mathrm{H}-\mathrm{I} 7 \mathrm{~d} 5$ & $7.764(6)$ \\
\hline 4415.580 & 52 & 22640.72 & 0.006 & 61493.77 & $6-38853.02$ & 6 & $17 \mathrm{p} 3-$ & \\
\hline 4386.846 & 80 & 22789.02 & 0.008 & 5081.77 & $4-27870.83$ & 5 & 3F-I6d5 & $7.373(6)$ \\
\hline 4375.880 & 47 & 22846.13 & 0.026 & 61699.28 & $7-38853.02$ & 6 & I7p3- & \\
\hline 4362.230 & 70 & 22917.61 & 0.030 & 61699.28 & $7-38781.51$ & 6 & $17 \mathrm{p} 3-$ & \\
\hline 4362.014 & 80 & 22918.75 & 0.006 & 10785.51 & $4-33704.29$ & 5 & $3 \mathrm{H}-\mathrm{d}$ & \\
\hline 4356.549 & 80 & 22947.50 & 0.000 & 13219.80 & $2-36167.30$ & 3 & $3 F-d$ & $5.371(6)$ \\
\hline 4348.926 & 52 & 22987.72 & 0.006 & 55547.30 & $7-32559.55$ & 7 & I7p1-I5d5 & \\
\hline 4341.734 & 80 & 23025.80 & 0.004 & 6969.80 & $5-29995.62$ & 5 & $3 \mathrm{H}-\mathrm{I} 5 \mathrm{~d} 3$ & $4.799(6)$ \\
\hline 4338.234 & 80 & 23044.38 & 0.000 & 6969.80 & $5-30014.18$ & 6 & $3 \mathrm{H}-\mathrm{I} 5 \mathrm{~s} 1$ & $8.674(5)$ \\
\hline 4290.113 & 80 & 23302.85 & 0.007 & 0.00 & $6-23302.89$ & 7 & $3 \mathrm{H}-\mathrm{I} 7 \mathrm{~d} 5$ & $1.671(7)^{b}$ \\
\hline 4288.191 & 80 & 23313.30 & -0.002 & 6969.80 & $5-30282.09$ & 6 & $3 \mathrm{H}-15 \mathrm{~d} 3$ & $2.381(6)$ \\
\hline 4284.687 & 46 & 23332.36 & -0.006 & 62598.14 & $6-39265.81$ & 5 & I6pl- & \\
\hline 4266.570 & 80 & 23431.43 & -0.004 & 12472.55 & $3-35903.96$ & 4 & $3 F-F 4 d$ & $1.162(6)$ \\
\hline 4219.151 & 48 & 23694.78 & -0.005 & 12472.55 & $3-36167.30$ & 3 & $3 F-d$ & \\
\hline 4210.203 & 38 & 23745.13 & -0.002 & 62598.14 & $6-38853.02$ & 6 & 16p1- & \\
\hline 4208.465 & 45 & 23754.94 & -0.018 & 62607.86 & $7-38853.02$ & 6 & $16 \mathrm{p} 1-$ & \\
\hline 4203.958 & 38 & 23780.41 & 0.002 & 6969.80 & $5-30750.22$ & 4 & $3 \mathrm{H}-\mathrm{d}$ & \\
\hline 4197.569 & 59 & 23816.60 & 0.005 & 62598.14 & $6-38781.51$ & 6 & I6p1- & \\
\hline 4123.447 & 80 & 24244.72 & 0.000 & 6969.80 & $5-31214.52$ & 5 & $3 \mathrm{H}-\mathrm{d}$ & $2.120(6)$ \\
\hline
\end{tabular}


Table I. Continued

\begin{tabular}{|c|c|c|c|c|c|c|c|c|}
\hline (1) & (2) & (3) & (4) & (5) & (6) $(7)$ & (8) & (9) & (10) \\
\hline 4099.277 & 48 & 24387.67 & -0.007 & 5081.77 & $4-29469.40$ & 4 & $3 F-I d$ & \\
\hline 4088.566 & 48 & 24451.55 & -0.012 & 55547.30 & $7-31095.82$ & 6 & I7p1-I4d3 & \\
\hline 4065.037 & 80 & 24593.08 & -0.002 & 13219.80 & $2-37812.87$ & 3 & $3 F-d$ & $1.611(6)$ \\
\hline 4012.691 & 70 & 24913.89 & -0.007 & 5081.77 & $4-29995.62$ & 5 & $3 F-15 d 3$ & $9.364(5)$ \\
\hline 4009.709 & 80 & 24932.42 & 0.002 & 6969.80 & $5-31902.23$ & 4 & $3 \mathrm{H}-\mathrm{I} 4 \mathrm{~s} 1$ & $1.061(6)$ \\
\hline 3978.309 & 39 & 25129.20 & -0.003 & 61699.28 & $7-36570.10$ & 6 & I7p3-I4d5 & \\
\hline 3977.306 & 43 & 25135.54 & 0.005 & 12472.55 & $3-37608.12$ & 4 & $3 F-d$ & \\
\hline 3972.981 & 37 & 25162.90 & 0.010 & 61493.77 & $6-36330.81$ & 5 & I7p3-d & \\
\hline 3963.457 & 70 & 25223.36 & 0.005 & 6969.80 & 5-32193.19 & 5 & $3 \mathrm{H}-\mathrm{I} 4 \mathrm{~s} 1$ & $1.067(6)$ \\
\hline 3962.862 & 80 & 25227.15 & 0.002 & 6969.80 & $5-32196.96$ & 4 & $3 \mathrm{H}-\mathrm{d}$ & $5.931(6)$ \\
\hline 3962.862 & 80 & 25227.15 & 0.010 & 71779.39 & $5-46552.18$ & 5 & I5p3-?d & \\
\hline 3945.166 & 80 & 25340.30 & 0.003 & 12472.55 & $3-37812.87$ & 3 & $3 F-d$ & $1.568(6)$ \\
\hline 3944.563 & 48 & 25344.18 & -0.002 & 13219.80 & $2-38563.97$ & 2 & $3 F-? d$ & \\
\hline 3943.009 & 38 & 25354.16 & 0.012 & 65934.64 & $5-40580.40$ & 4 & I5p1- & \\
\hline $3938.717 \mathrm{~T}$ & & 25381.79 & & 10785.51 & $4-36167.30$ & 3 & $3 \mathrm{H}-\mathrm{d}$ & $4.376(6)^{\circ}$ \\
\hline 3913.504 & 80 & 25545.31 & -0.001 & 10785.51 & $4-36330.81$ & 5 & $3 \mathrm{H}-\mathrm{d}$ & $6.797(6)$ \\
\hline 3898.347 & 70 & 25644.63 & -0.009 & 6969.80 & $5-32614.37$ & 5 & $3 \mathrm{H}-\mathrm{d}$ & $2.344(6)$ \\
\hline 3889.267 & 54 & 25704.50 & 0.000 & 13219.80 & $2-38924.30$ & 3 & $3 F-d$ & $2.115(6)$ \\
\hline 3854.499 & 21 & 25936.36 & -0.006 & 55547.30 & $7-29610.99$ & 7 & I7p1-I6d5 & 2 \\
\hline 3853.585 & 80 & 25942.51 & 0.004 & 62598.14 & $6-36655.60$ & 5 & I6p1-d5 & \\
\hline 3835.724 & 59 & 26063.30 & 0.000 & 6969.80 & $5-33033.10$ & 4 & $3 \mathrm{H}-\mathrm{d}$ & $9.238(5)$ \\
\hline 3831.596 & 45 & 26091.38 & 0.006 & 12472.55 & $3-38563.97$ & 2 & $3 F-? d$ & \\
\hline 3825.531 & 48 & 26132.70 & 0.000 & 5081.77 & $4-31214.52$ & 5 & $3 F-d$ & \\
\hline 3816.765 & 80 & 26192.76 & -0.009 & 0.00 & $6-26192.70$ & 5 & $3 \mathrm{H}-\mathrm{I} 6 \mathrm{~d} 3$ & $8.282(6)$ \\
\hline 3812.552 & 70 & 26221.71 & 0.003 & 6969.80 & 5-33191.53 & 6 & $3 \mathrm{H}-\mathrm{d}$ & \\
\hline 3805.935 & 48 & 26267.30 & -0.003 & 65934.64 & $5-39667.36$ & 4 & I5p1-F3d3 & \\
\hline 3805.935 & 48 & 26267.30 & 0.004 & 62598.14 & $6-36330.81$ & 5 & I6p1-d & \\
\hline 3779.402 & 70 & 26451.70 & 0.007 & 12472.55 & $3-38924.30$ & 3 & $3 F-d$ & $1.550(6)$ \\
\hline 3761.163 & 80 & 26579.97 & 0.000 & 0.00 & $6-26579.97$ & 7 & $3 \mathrm{H}-\mathrm{I} 6 \mathrm{~d} 3$ & $1.791(7)^{d}$ \\
\hline 3739.422 & 80 & 26734.50 & -0.001 & 6969.80 & 5-33704.29 & 5 & $3 \mathrm{H}-\mathrm{d}$ & $8.564(6)$ \\
\hline 3738.434 & 48 & 26471.57 & -0.006 & 62598.14 & $6-35856.62$ & 6 & 16p1-d & \\
\hline 3734.508 & 70 & 26769.68 & -0.017 & 55547.30 & $7-28777.74$ & 6 & $17 p 1-16 d 5$ & \\
\hline 3727.130 & 80 & 26822.67 & -0.008 & 10785.51 & $4-37608.12$ & 4 & $3 \mathrm{H}-\mathrm{d}$ & $2.270(7)$ \\
\hline 3715.666 & 80 & 26905.42 & -0.004 & 6969.80 & $5-33875.19$ & 6 & $3 \mathrm{H}-\mathrm{I} 5 \mathrm{~d} 5$ & $5.908(6)$ \\
\hline 3704.931 & 38 & 26983.38 & 0.007 & 73920.66 & $5-46937.23$ & 4 & I4p3- & \\
\hline 3703.765 & 48 & 26991.87 & 0.004 & 55547.30 & $7-28555.40$ & 8 & $17 p 1-16 d 5$ & \\
\hline 3698.901 & 42 & 27027.37 & -0.001 & 10785.51 & $4-37812.87$ & 3 & $3 \mathrm{H}-\mathrm{d}$ & \\
\hline 3687.443 & 48 & 27111.42 & 0.000 & 5081.77 & 4-32193.19 & 5 & $3 F-14 s 1$ & \\
\hline 3684.999 & 48 & 27129.33 & -0.007 & 67986.38 & $5-40857.10$ & 5 & I6p3-?d & \\
\hline 3676.510 & 42 & 27191.97 & -0.001 & 74129.19 & $4-46937.23$ & 4 & I4p3- & \\
\hline 3658.065 & 59 & 27329.07 & -0.008 & 68186.11 & $6-40857.10$ & 5 & I6p3-?d & \\
\hline 3639.303 & 43 & 27469.96 & -0.005 & 56025.40 & $8-28555.40$ & 8 & I7p1-I6d5 & \\
\hline $3638.977 \mathrm{~T}$ & & 27472.46 & & 0.00 & $6-27472.42$ & 6 & $3 \mathrm{H}-\mathrm{I} 6 \mathrm{~d} 3$ & $3.711(7)^{c}$ \\
\hline 3619.718 & 43 & 27618.58 & 0.000 & 61493.77 & $6-33875.19$ & 6 & $17 \mathrm{p} 3-15 \mathrm{~d} 5$ & \\
\hline 3592.978 & 59 & 27824.13 & -0.005 & 61699.28 & $7-33875.19$ & 6 & $17 \mathrm{p} 3-\mathrm{I} 5 \mathrm{~d} 5$ & \\
\hline 3586.954 & 80 & 27870.85 & -0.003 & 0.00 & $6-27870.83$ & 5 & $3 \mathrm{H}-16 \mathrm{~d} 5$ & $6.860(6)$ \\
\hline 3578.693 & 45 & 27935.19 & -0.003 & 12472.55 & $3-40407.72$ & 3 & $3 F-d$ & \\
\hline 3501.161 & 80 & 28553.78 & -0.017 & 18383.59 & $4-46937.23$ & 4 & 1G- & \\
\hline 3501.161 & 80 & 28553.78 & 0.001 & 56025.40 & $8-27471.61$ & 9 & I7p1-I6d5 & \\
\hline 3492.747 & 48 & 28622.57 & -0.006 & 5081.77 & $4-33704.29$ & 5 & $3 F-d$ & \\
\hline 3487.108 & 41 & 28668.86 & 0.008 & 75221.11 & $6-46552.18$ & 5 & H5p1-?d & \\
\hline 3480.525 & 80 & 28723.07 & -0.015 & 62598.14 & $6-33875.19$ & 6 & I6p1-I5d5 & \\
\hline 3479.377 & 80 & 28732.56 & 0.013 & 62607.86 & $7-33875.19$ & 6 & I6p1-I5d5 & \\
\hline $3473.914 \mathrm{~T}$ & & 28777.74 & & 0.00 & 6-28777.74 & 6 & $3 \mathrm{H}-\mathrm{I} 6 \mathrm{~d} 5$ & $3.082(7)^{f}$ \\
\hline $3469.007 \mathrm{~T}$ & & 28818.44 & & 0.00 & 6-28818.44 & 7 & $3 \mathrm{H}-15 \mathrm{~d} 3$ & $1.437(7)$ \\
\hline 3461.675 & 42 & 28879.48 & -0.010 & 61493.77 & $6-32614.37$ & 5 & I7p3-d & \\
\hline 3460.796 & 80 & 28886.82 & 0.000 & 6969.80 & $5-35856.62$ & 6 & $3 \mathrm{H}-\mathrm{d}$ & \\
\hline 3455.125 & 48 & 28934.23 & -0.008 & 6969.80 & $5-35903.96$ & 4 & $3 \mathrm{H}-\mathrm{F} 4 \mathrm{~d}$ & $7.910(6)$ \\
\hline 3455.125 & 48 & 28934.23 & -0.001 & 61493.77 & $6-32559.55$ & 7 & I7p3-ISd5 & \\
\hline 3451.179 & 59 & 28967.30 & 0.004 & 55547.30 & $7-26579.97$ & 7 & I7p1-I6d3 & \\
\hline 3423.101 & 42 & 29204.90 & -0.004 & 67986.38 & $5-38781.51$ & 6 & I6p3- & \\
\hline 3414.435 & 59 & 29279.03 & 0.001 & 65934.64 & $5-36655.60$ & 5 & $15 \mathrm{p} 1-\mathrm{d} 5$ & \\
\hline 3404.896 & 59 & 29361.05 & -0.005 & 6969.80 & $5-36330.81$ & 5 & $3 \mathrm{H}-\mathrm{d}$ & $1.691(6)$ \\
\hline 3395.673 & 43 & 29440.79 & -0.001 & 66077.65 & $6-36636.87$ & 7 & I5p1-F4d5 & \\
\hline 3395.247 & 59 & 29444.49 & 0.002 & 55547.30 & $7-26102.79$ & 7 & I7p1-I6s1 & \\
\hline 3395.132 & 38 & 29445.49 & -0.006 & 56025.40 & $8-26579.97$ & 7 & $17 \mathrm{p} 1-\mathrm{I} 6 \mathrm{~d} 3$ & \\
\hline 3391.248 & 47 & 29479.21 & -0.002 & 68332.21 & $5-38853.02$ & 6 & I $4 \mathrm{p} 1-$ & \\
\hline 3377.373 & 46 & 29600.31 & -0.001 & 6969.80 & $5-36570.10$ & 6 & $3 \mathrm{H}-\mathrm{I} 4 \mathrm{~d} 5$ & $1.566(6)$ \\
\hline 3376.160 & 59 & 29610.95 & 0.005 & 0.00 & 6-29610.99 & 7 & $3 \mathrm{H}-\mathrm{I} 6 \mathrm{~d} 5$ & $1.872(6)$ \\
\hline 3367.631 & 80 & 29685.94 & -0.016 & 6969.80 & $5-36655.60$ & 5 & $3 \mathrm{H}-\mathrm{d} 5$ & $2.303(7)$ \\
\hline 3360.729 & 39 & 29746.90 & -0.007 & 66077.65 & $6-36330.81$ & 5 & I5p1-d & \\
\hline
\end{tabular}


Table I. Continued

\begin{tabular}{|c|c|c|c|c|c|c|c|c|}
\hline (1) & (2) & (3) & (4) & (5) & (6) $(7)$ & (8) & (9) & (10) \\
\hline 3353.106 & 41 & 29814.52 & 0.000 & 76751.75 & $5-46937.23$ & 4 & F4p3- & \\
\hline 3340.992 & 43 & 29922.62 & -0.001 & 56025.40 & $8-26102.79$ & 7 & $\mathrm{I} 7 \mathrm{p} 1-\mathrm{I} 6 \mathrm{~s} 1$ & \\
\hline 3332.862 & 39 & 29995.61 & 0.001 & 0.00 & $6-29995.62$ & 5 & $3 \mathrm{H}-\mathrm{I} 5 \mathrm{~d} 3$ & \\
\hline 3327.014 & 80 & 30048.34 & -0.003 & 62607.86 & $7-32559.55$ & 7 & I6p1-I5d5 & \\
\hline 3325.168 & 45 & 30065.02 & 0.006 & 55547.30 & $7-25482.23$ & 8 & I7p1-I6d3 & \\
\hline 3307.998 & 80 & 30221.07 & -0.004 & 66077.65 & $6-35856.62$ & 6 & I5p1-d & \\
\hline 3301.638 & 38 & 30279.28 & -0.003 & 61493.77 & $6-31214.52$ & 5 & I7p3-d & \\
\hline 3301.228 & NS & 30283.04 & 0.005 & 0.00 & $6-30283.09$ & 6 & $3 \mathrm{H}-\mathrm{I} 5 \mathrm{~d} 3$ & $4.595(6)$ \\
\hline 3287.986 & 32 & 30405.00 & -0.005 & 62598.14 & $6-32193.19$ & 5 & I6p1-I4s1 & \\
\hline 3264.236 & 46 & 30626.21 & 0.004 & 68234.37 & $4-37608.12$ & 4 & 14p1-d & \\
\hline 3262.948 & 48 & 30638.30 & 0.002 & 6969.80 & $5-37608.12$ & 4 & $3 \mathrm{H}-\mathrm{d}$ & $4.217(6)$ \\
\hline 3243.477 & 80 & 30822.21 & -0.002 & 5081.77 & $4-35903.96$ & 4 & 3F-F4d & $1.020(7)$ \\
\hline 3234.645 & 42 & 30906.37 & 0.003 & 62607.86 & $7-31701.46$ & 8 & I6p1-I5d5 & \\
\hline 3214.950 & NS & 31095.70 & 0.012 & 0.00 & $6-31095.82$ & 6 & $3 \mathrm{H}-\mathrm{I} 4 \mathrm{~d} 3$ & $3.982(6)$ \\
\hline 3175.743 & NS & 31479.59 & 0.001 & 61493.77 & $6-30014.18$ & 6 & I7p3-I5s1 & \\
\hline 3173.454 & NS & 31502.29 & 0.003 & 62598.14 & $6-31095.8$ & 6 & $16 \mathrm{p} 1-14 \mathrm{~d} 3$ & \\
\hline 3172.470 & NS & 31512.06 & -0.002 & 62607.86 & $7-31095.2$ & 6 & $16 \mathrm{p} 1-\mathrm{I} 4 \mathrm{~d} 3$ & \\
\hline 3166.262 & 120 & 31573.85 & -0.002 & 5081.77 & $4-36655.60$ & 5 & $3 F-d 5$ & $2.802(7)$ \\
\hline 3135.536 & 41 & 31883.24 & -0.002 & 6969.80 & $5-38853.02$ & 6 & $3 \mathrm{H}-$ & \\
\hline 3117.558 & 48 & 32067.09 & -0.002 & 68234.37 & $4-36167.30$ & 3 & I4p1-d & $9.151(7)$ \\
\hline 3100.400 & 110 & 32244.48 & -0.007 & 55547.30 & $7-23302.89$ & 7 & I7p1-I7d5 & $9.556(7)$ \\
\hline 3095.458 & 38 & 32296.02 & -0.001 & 6969.80 & $5-39265.81$ & 5 & $3 \mathrm{H}-$ & \\
\hline 3093.632 & 39 & 32315.08 & -0.003 & 62598.14 & $6-30283.09$ & 6 & I6p1-I5d3 & \\
\hline 3092.701 & 59 & 32324.81 & -0.004 & 62607.86 & $7-30283.09$ & 6 & I6p1-I5d3 & \\
\hline 3073.537 & 48 & 32526.35 & 0.000 & 5081.77 & $4-37608.12$ & 4 & $3 F-d$ & $1.617(7)$ \\
\hline 3070.402 & 48 & 32559.56 & -.001 & 0.00 & $6-32559.55$ & 7 & $3 \mathrm{H}-\mathrm{I} 5 \mathrm{~d} 5$ & $1.473(7)$ \\
\hline 3055.710 & 48 & 32716.10 & -0.007 & 61493.77 & $6-28777.74$ & 6 & I7p3-I6d5 & \\
\hline 3055.106 & 80 & 32722.58 & -0.007 & 56025.40 & $8-23302.89$ & 7 & I7p1-I7d5 & \\
\hline 3038.491 & 39 & 32901.50 & 0.004 & 65934.64 & $5-33033.10$ & 4 & $15 p 1-d$ & \\
\hline 3036.640 & 70 & 32921.56 & -0.002 & 61699.28 & $7-28777.74$ & 6 & I7p3-I6d5 & $7.417(7)$ \\
\hline 3022.655 & 41 & 33073.86 & 0.001 & 56025.40 & $8-22951.53$ & 8 & I7p1-I7d5 & \\
\hline 2971.227 & 41 & 33646.30 & 0.004 & 71459.21 & $4-37812.87$ & 3 & I5p3-d & $8.846(7)$ \\
\hline 2963.186 & 43 & 33737.60 & 0.007 & 65934.64 & $5-32196.96$ & 4 & $15 \mathrm{p} 1-\mathrm{d}$ & $8.181(7)$ \\
\hline 2958.644 & 47 & 33789.40 & 0.002 & 62607.86 & $7-28818.44$ & 7 & I6p1-I5d3 & $8.194(7)$ \\
\hline 2955.935 & 46 & 33820.36 & 0.003 & 62598.14 & $6-28777.74$ & 6 & I6p1-I6d5 & \\
\hline 2920.825 & NS & 34226.88 & -0.006 & 61699.28 & $7-27472.46$ & 6 & $17 p 3-16 d 3$ & \\
\hline 2916.116 & 41 & 34282.16 & -0.006 & 67986.38 & $5-33704.29$ & 5 & I6p3-d & $9.579(7)$ \\
\hline 2909.163 & 41 & 34364.08 & -0.006 & 75221.11 & $6-40857.10$ & 5 & H5p1-?d & \\
\hline 2909.163 & 41 & 34364.08 & -0.001 & 74944.47 & $5-40580.40$ & 4 & H5p1- & $1.147(8)$ \\
\hline 2906.163 & 38 & 34399.56 & 0.000 & 71055.16 & $4-36655.60$ & 5 & F4p1-d & $9.388(7)$ \\
\hline 2900.662 & 43 & 34464.79 & -0.009 & 12472.55 & $3-46937.23$ & 4 & $3 F-$ & \\
\hline 2890.527 & 46 & 34585.62 & -0.003 & 5081.77 & $4-39667.36$ & 4 & $3 F-F 3 d 3$ & $3.983(7)$ \\
\hline 2878.733 & 100 & 34727.31 & 0.000 & 62598.14 & $6-27870.83$ & 5 & I6p1-I6d5 & $2.197(8)$ \\
\hline 2869.517 & 40 & 34838.84 & -0.002 & 65934.64 & $5-31095.82$ & 6 & I5p1-I4d3 & $8.238(7)$ \\
\hline 2849.629 & 48 & 35081.98 & -0.004 & 61493.77 & $6-26411.84$ & 6 & I7p3-I6s 1 & $1.592(8)$ \\
\hline 2846.592 & 39 & 35119.40 & -0.007 & 61699.28 & $7-26579.97$ & 7 & $17 \mathrm{p} 3-16 \mathrm{~d} 3$ & \\
\hline 2846.080 & 120 & 35125.72 & -0.003 & 62598.14 & $6-27472.46$ & 6 & I6p1-I6d3 & $7.663(8)$ \\
\hline 2845.293 & 120 & 35135.44 & -0.003 & 62607.86 & $7-27472.46$ & 6 & I6p1-I6d3 & $3.378(8)$ \\
\hline 2844.988 & 45 & 35139.21 & -0.005 & 73920.66 & $5-38781.51$ & 6 & I4p3- & \\
\hline 2840.591 & 47 & 35193.60 & -0.008 & 71050.12 & $5-35856.62$ & 6 & F4p1-d & $2.983(8)$ \\
\hline 2833.031 & 70 & 35287.51 & -0.006 & 61699.28 & $7-26411.84$ & 6 & I7p3-I6s 1 & $3.414(8)$ \\
\hline 2832.674 & 45 & 35291.95 & -0.003 & 71459.21 & $4-36167.30$ & 3 & I5p3-d & $2.163(8)$ \\
\hline 2830.340 & 100 & 35321.05 & 0.002 & 55547.30 & $7-20226.22$ & 7 & $17 \mathrm{p} 1-17 \mathrm{~s} 1$ & $9.694(8)$ \\
\hline 2824.747 & 110 & 35390.99 & -0.001 & 61493.77 & $6-26102.79$ & 7 & I7p3-I6s1 & $7.489(8)$ \\
\hline 2816.182 & 43 & 35498.61 & 0.001 & 5081.77 & $4-40580.40$ & 4 & $3 F-$ & $1.846(7)$ \\
\hline 2811.697 & 41 & 35555.24 & 0.001 & 71459.21 & $4-35903.96$ & 4 & I5p3-F4d & \\
\hline 2808.634 & 46 & 35594.02 & -0.006 & 76174.34 & $3-40580.40$ & 4 & F3p1- & \\
\hline 2808.437 & 47 & 35596.51 & -0.002 & 61699.28 & $7-26102.79$ & 7 & I7p3-I6s 1 & $1.573(8)$ \\
\hline 2806.584 & 120 & 35620.01 & -0.001 & 68234.37 & $4-32614.37$ & 5 & I4p1-d & $9.538(8)$ \\
\hline 2805.869 & 120 & 35629.08 & -0.003 & 55547.30 & $7-19918.26$ & 8 & I7p1-I7d3 & $1.193(9)$ \\
\hline 2804.098 & 90 & 35651.58 & -0.002 & 65934.64 & $5-30283.09$ & 6 & I5p1-I5d3 & $6.315(8)$ \\
\hline 2798.896 & 43 & 35717.85 & -0.001 & 68332.21 & $5-32614.37$ & 5 & I4p1-d & $1.502(8)$ \\
\hline 2795.907 & 48 & 35756.02 & -0.003 & 71050.12 & $5-35294.14$ & 4 & F4p1-F4s1 & $1.345(9)$ \\
\hline 2795.517 & 46 & 35761.02 & 0.000 & 71055.16 & $4-35294.14$ & 4 & $\mathrm{~F} 4 \mathrm{p} 1-\mathrm{F} 4 \mathrm{~s} 1$ & $6.740(8)$ \\
\hline 2795.081 & 41 & 35766.60 & 0.002 & 76174.34 & $3-40407.72$ & 3 & F3p1-d & $4.462(8)$ \\
\hline 2795.081 & 41 & 35766.60 & 0.006 & 10785.51 & $4-46552.18$ & 5 & $3 \mathrm{H}-\mathrm{dd}$ & \\
\hline 2793.296 & 41 & 35789.45 & -0.002 & 67986.38 & $5-32196.96$ & 4 & $16 \mathrm{p} 3-\mathrm{d}$ & $1.287(8)$ \\
\hline 2792.900 & 42 & 35794.52 & 0.003 & 66077.65 & 6-30283.09 & 6 & 15p1-15d3 & $1.471(8)$ \\
\hline 2792.539 & 59 & 35799.15 & 0.002 & 56025.40 & $8-20226.22$ & 7 & I7p1-17s1 & $3.408(9)$ \\
\hline 2788.065 & 45 & 35856.59 & 0.002 & 0.00 & $6-35856.62$ & 6 & $3 \mathrm{H}-\mathrm{d}$ & $4.689(7)$ \\
\hline 2783.109 & 48 & 35920.45 & 0.001 & 65934.64 & $5-30014.18$ & 6 & $\mathrm{I} 5 \mathrm{p} 1-\mathrm{I} 5 \mathrm{~s} 1$ & $2.003(9)$ \\
\hline
\end{tabular}


Table I. Continued

\begin{tabular}{|c|c|c|c|c|c|c|c|c|}
\hline (1) & (2) & (3) & (4) & (5) & (6) $(7)$ & (8) & (9) & (10) \\
\hline 2782.440 & 38 & 35929.07 & 0.002 & 71785.72 & $6-35856.62$ & 6 & I5p3-d & \\
\hline 2781.669 & 46 & 35939.03 & -0.001 & 65934.64 & $5-29995.62$ & 5 & I5p1-I5d3 & $7.705(8)$ \\
\hline 2780.405 & 47 & 35955.37 & -0.005 & 75221.11 & $6-39265.81$ & 5 & H5p1- & \\
\hline 2776.347 & 48 & 36007.93 & -0.003 & 76174.34 & $3-40166.45$ & 4 & $\mathrm{~F} 3 \mathrm{p} 1-\mathrm{F} 3 \mathrm{~s} 1$ & $1.346(9)$ \\
\hline 2775.556 & 48 & 36018.18 & -.001 & 62598.14 & $6-26579.97$ & 7 & I6p1-I6d3 & $4.072(8)$ \\
\hline 2774.805 & 48 & 36027.93 & -0.003 & 62607.86 & $7-26579.97$ & 7 & I6p1-I6d3 & $1.168(9)$ \\
\hline 2773.785 & 48 & 36041.18 & 0.000 & 68234.37 & $4-32193.19$ & 5 & $\mathrm{I} 4 \mathrm{p} 1-\mathrm{I} 4 \mathrm{~s} 1$ & $1.184(9)$ \\
\hline 2772.073 & 48 & 36063.44 & 0.002 & 66077.65 & $6-30014.18$ & 6 & I5p1-I5s1 & $1.549(9)$ \\
\hline 2771.361 & 42 & 36072.71 & -0.006 & 76480.35 & $4-40407.72$ & 3 & F3p1-d & $8.871(8)$ \\
\hline 2770.643 & 41 & 36082.05 & -0.001 & 66077.65 & 6-29995.62 & 5 & I5p1-I5d3 & \\
\hline 2770.481 & 39 & 36084.16 & -0.001 & 67986.38 & $5-31902.23$ & 4 & I6p3-I4s1 & $1.757(8)$ \\
\hline 2768.715 & 48 & 36107.17 & -0.002 & 56025.40 & $8-19918.26$ & 8 & I7p1-I7d3 & $7.895(8)$ \\
\hline 2767.360 & 48 & 36124.85 & -0.005 & 71050.12 & $5-34925.33$ & 5 & F4p1-F4s1 & $1.489(9)$ \\
\hline 2767.106 & 48 & 36128.17 & -0.001 & 65934.64 & 5-29806.48 & 5 & I5p1-I5s1 & $5.432(8)$ \\
\hline 2766.976 & 48 & 36129.87 & -0.003 & 71055.16 & $4-34925.33$ & 5 & F4p1-F4s1 & $1.521(9)$ \\
\hline 2766.562 & 48 & 36135.28 & -0.002 & 68332.21 & 5-32196.96 & 4 & I4p1-d & $9.003(8)$ \\
\hline 2766.273 & 48 & 36139.05 & -0.002 & 68332.21 & $5-32193.19$ & 5 & I4p1-I4s1 & $1.011(9)$ \\
\hline 2764.442 & 48 & 36162.98 & -0.001 & 74944.47 & $5-38781.51$ & 6 & H5p1- & $2.508(9)$ \\
\hline 2764.281 & 39 & 36165.09 & -0.002 & 71459.21 & $4-35294.14$ & 4 & I5p3-F4s1 & $2.779(8)$ \\
\hline 2762.659 & 48 & 36186.32 & -0.001 & 62598.14 & $6-26411.84$ & 6 & I6p1-I6s1 & $8.357(8)$ \\
\hline 2761.915 & 59 & 36196.07 & -0.004 & 62607.86 & $7-26411.84$ & 6 & I6p1-I6s1 & $2.844(9)$ \\
\hline 2759.226 & 70 & 36231.34 & 0.000 & 55547.30 & $7-19315.96$ & 8 & I7p1-I7s1 & 3.587 (9) \\
\hline 2756.196 & 70 & 36271.17 & 0.000 & 66077.65 & 6-29806.48 & 5 & I5p1-I5s1 & $2.696(9)$ \\
\hline 2752.957 & 47 & 36313.84 & 0.004 & 76480.35 & 4-40166.45 & 4 & $F 3 p 1-F 3 s 1$ & $7.823(8)$ \\
\hline 2751.567 & 48 & 36332.18 & -0.003 & 68234.4 & $4-31902.23$ & 4 & I4p1-I4s 1 & $1.075(9)$ \\
\hline 2748.848 & 48 & 36368.12 & -0.002 & 75221.11 & $6-38853.02$ & 6 & H5p1- & $8.733(8)$ \\
\hline 2746.032 & 48 & 36405.42 & 0.002 & 62598.14 & $6-26192.70$ & 5 & I6p1-I6d3 & $1.084(9)$ \\
\hline 2744.183 & 48 & 36429.95 & 0.002 & 68332.21 & $5-31902.23$ & 4 & I4p1-I4s1 & $1.485(9)$ \\
\hline 2743.456 & 47 & 36439.60 & 0.000 & 75221.11 & $6-38781.51$ & 6 & H5p1- & $1.574(9)$ \\
\hline 2741.417 & 46 & 36466.70 & -0.003 & 66077.65 & 6-29610.99 & 7 & I5p1-I6d5 & $3.559(8)$ \\
\hline 2740.018 & 41 & 36485.32 & -0.005 & 71779.39 & $5-35294.14$ & 4 & I5p3-F4s1 & \\
\hline 2739.266 & 70 & 36495.33 & 0.002 & 62598.14 & $6-26102.79$ & 7 & I6p1-I6s1 & 2.897 (9) \\
\hline 2738.534 & 70 & 36505.08 & -0.001 & 62607.86 & $7-26102.79$ & 7 & I6p1-I6s 1 & $1.574(9)$ \\
\hline 2737.334 & 42 & 36521.09 & -0.001 & 74129.19 & $4-37608.12$ & 4 & I4p3-d & \\
\hline 2736.375 & 43 & 36533.88 & 0.000 & 71459.21 & $4-34925.33$ & 5 & I5p3-F4s1 & \\
\hline 2727.287 & 42 & 36655.62 & -0.001 & 0.00 & $6-36655.60$ & 5 & $3 \mathrm{H}-\mathrm{d} 5$ & $3.140(7)$ \\
\hline 2723.288 & 120 & 36709.44 & 0.000 & 56025.40 & $8-19315.96$ & 8 & I7p1-I7s1 & $2.839(9)$ \\
\hline 2715.628 & 48 & 36812.99 & 0.000 & 76480.35 & 4-39667.36 & 4 & F3p1-F3d3 & $8.490(8)$ \\
\hline 2712.601 & 38 & 36854.07 & -0.001 & 71779.39 & $5-34925.33$ & 5 & $15 p 3-F 4 s 1$ & $2.164(8)$ \\
\hline 2703.987 & 41 & 36971.46 & 0.009 & 68186.11 & $6-31214.52$ & 5 & I6p3-d & \\
\hline 2700.456 & 42 & 37019.81 & 0.003 & 68234.37 & $4-31214.52$ & 5 & I4p $1-d$ & $2.511(8)$ \\
\hline 2698.359 & 120 & 37048.57 & 0.001 & 56025.40 & $8-18976.82$ & 9 & I7p1-I7d3 & 2.477 (9) \\
\hline 2693.334 & 80 & 37117.69 & 0.000 & 68332.21 & $5-31214.52$ & 5 & I4p1-d & $8.708(8)$ \\
\hline 2692.766 & 120 & 37125.52 & 0.008 & 62607.86 & $7-25482.23$ & 8 & I6p1-I6d3 & $2.113(9)$ \\
\hline 2690.867 & 39 & 37151.71 & -0.010 & 77318.02 & $3-40166.45$ & 4 & F4p3-F3s1 & \\
\hline 2684.747 & 100 & 37236.40 & -0.001 & 68332.21 & $5-31095.82$ & 6 & $\mathrm{I} 4 \mathrm{p} 1-\mathrm{I} 4 \mathrm{~d} 3$ & $1.552(9)$ \\
\hline 2683.100 & 100 & 37259.26 & -0.004 & 66077.65 & $6-28818.44$ & 7 & $15 p 1-15 d 3$ & $1.321(9)$ \\
\hline 2682.684 & 43 & 37265.04 & 0.001 & 73920.66 & $5-36655.60$ & 5 & $14 \mathrm{p3}-\mathrm{d} 5$ & $6.365(8)$ \\
\hline 2680.172 & 48 & 37299.96 & -0.004 & 66077.65 & $6-28777.74$ & 6 & I5p1-I6d5 & $4.964(8)$ \\
\hline 2666.872 & 38 & 37485.97 & -0.002 & 76751.75 & $5-39265.81$ & 5 & F4p3- & \\
\hline 2651.502 & 43 & 37703.25 & 0.003 & 67986.38 & $5-30283.09$ & 6 & I6p3-I5d3 & $4.478(8)$ \\
\hline 2647.872 & 38 & 37754.93 & -0.001 & 71459.21 & $4-33704.29$ & 5 & I5p3-d & $3.545(8)$ \\
\hline 2640.621 & 48 & 37858.60 & -0.001 & 71050.12 & $5-33191.53$ & 6 & F4p1-d & $9.064(8)$ \\
\hline 2639.858 & 47 & 37869.55 & -0.002 & 71055.16 & 4-33185.64 & 3 & F4p1-F4d3 & $6.010(8)$ \\
\hline 2637.778 & 80 & 37899.40 & 0.000 & 55547.30 & $7-17647.90$ & 7 & $\mathrm{I7p} 1-\mathrm{I} 7 \mathrm{~d} 3$ & $1.234(9)$ \\
\hline 2637.527 & 39 & 37903.02 & 0.000 & 68186.11 & $6-30283.09$ & 6 & I6p3-I5d3 & \\
\hline 2637.444 & 48 & 37904.20 & 0.000 & 71779.39 & $5-33875.19$ & 6 & I5p3-I5d5 & $7.099(8)$ \\
\hline 2637.007 & 48 & 37910.48 & 0.004 & 71785.72 & $6-33875.19$ & 6 & I5p3-I5d5 & $8.443(8)$ \\
\hline 2633.440 & 39 & 37961.83 & -0.013 & 10785.51 & $4-48747.15$ & 5 & $3 \mathrm{H}-? \mathrm{~d}$ & $2.558(7)$ \\
\hline 2633.440 & 39 & 37961.83 & 0.004 & 74129.19 & $4-36167.30$ & 3 & I4p3-d & $3.886(8)$ \\
\hline 2632.861 & 41 & 37970.18 & 0.004 & 76751.75 & $5-38781.51$ & 6 & F4p3- & \\
\hline 2631.439 & 42 & 37990.70 & 0.004 & 67986.38 & $5-29995.62$ & 5 & $\mathrm{I} 6 \mathrm{p} 3-\mathrm{I} 5 \mathrm{~d} 3$ & $3.985(8)$ \\
\hline 2627.018 & 42 & 38054.63 & 0.001 & 76978.95 & 4-38924.30 & 3 & F4p3-d & $2.191(8)$ \\
\hline 2626.377 & 59 & 38063.91 & -0.007 & 65934.64 & $5-27870.83$ & 5 & I5p1-I6d5 & $2.776(8)$ \\
\hline 2626.377 & 59 & 38063.91 & 0.009 & 73920.66 & $5-35856.62$ & 6 & I4p3-d & $9.813(8)$ \\
\hline 2625.604 & 45 & 38075.11 & -0.001 & 71779.39 & $5-33704.29$ & 5 & I5p3-d & $6.791(8)$ \\
\hline 2625.204 & 41 & 38080.91 & 0.000 & 61032.44 & $9-22951.53$ & 8 & $17 \mathrm{p} 3-17 \mathrm{~d} 5$ & $1.309(8)$ \\
\hline 2618.949 & 45 & 38171.86 & 0.005 & 68186.11 & $6-30014.18$ & 6 & I6p3-I5s1 & $3.664(8)$ \\
\hline 2618.402 & 40 & 38179.83 & 0.005 & 67986.38 & 5-29806.48 & 5 & I6p3-I5s1 & $1.497(8)$ \\
\hline 2617.649 & 59 & 38190.83 & 0.003 & 61493.77 & $6-23302.89$ & 7 & 17p3-17d5 & $4.677(8)$ \\
\hline 2614.536 & 47 & 38236.29 & 0.000 & 61539.18 & $8-23302.89$ & 7 & I7p3-I7d5 & $1.681(8)$ \\
\hline
\end{tabular}


Table I. Continued

\begin{tabular}{|c|c|c|c|c|c|c|c|c|}
\hline (1) & (2) & (3) & (4) & (5) & (6) $(7)$ & (8) & (9) & (10) \\
\hline 2606.010 & 42 & 38361.38 & 0.006 & 76174.34 & $3-37812.87$ & 3 & F3p1-d & $3.538(8)$ \\
\hline 2605.130 & 39 & 38374.34 & 0.002 & 74944.47 & $5-36570.10$ & 6 & H5p1-I4d5 & $2.913(8)$ \\
\hline 2604.916 & 80 & 38377.49 & 0.001 & 56025.40 & $8-17647.90$ & 7 & I7p1-I7d3 & $6.100(8)$ \\
\hline 2603.815 & 39 & 38393.72 & 0.000 & 77318.02 & $3-38924.30$ & 3 & F4p3-d & $3.558(8)$ \\
\hline 2603.633 & 60 & 38396.40 & -0.001 & 61699.28 & $7-23302.89$ & 7 & $17 \mathrm{p} 3-\mathrm{I} 7 \mathrm{~d} 5$ & $1.615(9)$ \\
\hline 2601.637 & 41 & 38425.86 & 0.018 & 71459.21 & $4-33033.10$ & 4 & I5p3-d & $2.766(8)$ \\
\hline 2600.965 & 59 & 38435.79 & -0.003 & 71050.12 & $5-32614.37$ & 5 & F4p1-d & $9.048(8)$ \\
\hline 2599.183 & 45 & 38462.13 & 0.003 & 65934.64 & $5-27472.46$ & 6 & I5p1-I6d3 & $2.499(8)$ \\
\hline 2598.404 & 59 & 38473.67 & 0.001 & 68084.68 & $7-29610.99$ & 7 & I6p3-I6d5 & $8.734(8)$ \\
\hline 2595.489 & 59 & 38516.87 & 0.007 & 67986.38 & $5-29469.40$ & 4 & I6p3-Id & $5.608(8)$ \\
\hline 2592.203 & 39 & 38565.69 & -0.012 & 75221.11 & $6-36655.60$ & 5 & H5pl-d5 & $2.923(8)$ \\
\hline 2591.839 & 90 & 38571.10 & -0.005 & 55547.30 & $7-16976.28$ & 6 & I7p1-I7d3 & $1.982(9)$ \\
\hline 2591.570 & 80 & 38575.12 & 0.000 & 68186.11 & $6-29610.99$ & 7 & I6p3-I6d5 & $1.062(9)$ \\
\hline 2590.970 & 46 & 38584.05 & 0.013 & 75221.11 & $6-36636.87$ & 7 & H5p1-F4d5 & \\
\hline 2590.727 & 80 & 38587.66 & -0.001 & 61539.18 & $8-22951.53$ & 8 & $17 \mathrm{p} 3-17 \mathrm{~d} 5$ & $1.452(9)$ \\
\hline 2590.727 & 80 & 38587.66 & 0.013 & 71779.39 & $5-33191.53$ & 6 & 15p3-d & \\
\hline 2589.548 & NS & 38605.23 & -0.002 & 66077.65 & $6-27472.46$ & 6 & I5p1-16d3 & $1.644(8)$ \\
\hline 2588.985 & 59 & 38613.62 & 0.003 & 74944.47 & $5-36300.81$ & 5 & H5p1-d & $6.474(8)$ \\
\hline 2588.124 & 42 & 38626.48 & 0.003 & 73920.66 & 5-35294.14 & 4 & I4p3-F4s1 & \\
\hline 2585.384 & 41 & 38667.40 & 0.005 & 76480.35 & $4-37812.87$ & 3 & F3p1-d & \\
\hline 2580.123 & 41 & 38746.24 & 0.003 & 71779.39 & $5-33033.10$ & 4 & I5p3 - d & $1.574(8)$ \\
\hline 2580.024 & 80 & 38747.74 & 0.001 & 61699.28 & $7-22951.53$ & 8 & I7p3-I7d5 & $1.379(9)$ \\
\hline 2579.603 & 59 & 38754.05 & 0.000 & 77318.02 & $3-38563.97$ & 2 & F4p3-?d & \\
\hline 2578.889 & 59 & 38764.79 & 0.012 & 68234.37 & $4-29469.40$ & 4 & I4p1-Id & $2.761(8)$ \\
\hline 2578.687 & 80 & 38767.82 & 0.009 & 68234.37 & $4-29466.42$ & 3 & I4p1-Id & $6.668(8)$ \\
\hline 2573.031 & 59 & 38853.04 & -0.001 & 0.00 & $6-38853.02$ & 6 & 3H- & \\
\hline 2573.031 & 59 & 38853.04 & 0.008 & 71050.12 & $5-32196.96$ & 4 & F4p1-d & \\
\hline 2572.441 & 48 & 38861.94 & 0.002 & 71055.16 & 4-32193.19 & 5 & F4p1-I4s1 & $2.248(8)$ \\
\hline 2571.194 & 47 & 38880.79 & 0.002 & 67699.20 & $8-28818.44$ & 7 & $16 \mathrm{p3}-15 \mathrm{~d} 3$ & $1.060(8)$ \\
\hline 2570.746 & 80 & 38887.56 & -0.002 & 61493.77 & $6-22606.12$ & 6 & $17 \mathrm{p} 3-17 \mathrm{~d} 5$ & $1.177(9)$ \\
\hline 2565.199 & 80 & 38971.65 & 0.005 & 71531.27 & $7-32559.55$ & 7 & $15 \mathrm{p} 3-15 \mathrm{~d} 5$ & $9.590(8)$ \\
\hline 2560.668 & 48 & 39040.61 & -0.006 & 74944.47 & $5-35903.96$ & 4 & H5p1-F4d & $1.707(8)$ \\
\hline 2557.227 & 80 & 39093.13 & -0.006 & 61699.28 & $7-22606.12$ & 6 & $17 \mathrm{p} 3-17 \mathrm{~d} 5$ & $7.999(8)$ \\
\hline 2553.920 & 80 & 39143.75 & -0.008 & 76751.75 & $5-37608.12$ & 4 & F4p3-d & \\
\hline 2553.920 & 80 & 39143.75 & 0.003 & 67699.20 & $8-28555.40$ & 8 & I6p3-I6d5 & $1.112(9)$ \\
\hline 2552.535 & 39 & 39164.99 & 0.002 & 71779.39 & $5-32614.37$ & 5 & $15 p 3-d$ & \\
\hline 2552.470 & 43 & 39165.98 & 0.006 & 76978.95 & $4-37812.87$ & 3 & F4p3-d & $2.003(8)$ \\
\hline 2550.008 & 45 & 39203.79 & 0.005 & 74129.19 & $4-34925.33$ & 5 & $\mathrm{I} 4 \mathrm{p} 3-\mathrm{F} 4 \mathrm{~s} 1$ & \\
\hline 2549.698 & 41 & 39208.57 & 0.005 & 67986.38 & $5-28777.74$ & 6 & I6p3-I6d5 & $1.495(8)$ \\
\hline 2548.557 & 80 & 39226.11 & 0.004 & 71785.72 & $6-32559.55$ & 7 & I5p3-I5d5 & $1.626(9)$ \\
\hline 2546.213 & 45 & 39262.23 & 0.001 & 71459.21 & 4-32196.96 & 4 & I5p3-d & \\
\hline 2545.953 & 80 & 39266.24 & 0.000 & 68084.68 & $7-28818.44$ & 7 & $16 \mathrm{p} 3-15 \mathrm{~d} 3$ & $5.974(8)$ \\
\hline 2544.673 & 80 & 39285.99 & 0.004 & 65934.64 & $5-26648.4$ & 4 & I5p1-I5d3 & $1.024(9)$ \\
\hline 2543.320 & 59 & 39306.89 & 0.003 & 68084.68 & $7-28777.76$ & 6 & I6p3-I6d5 & $3.831(8)$ \\
\hline 2540.909 & 80 & 39344.17 & 0.000 & 61032.44 & $9-21688.27$ & 9 & $17 p 3-17 d 5$ & $1.105(9)$ \\
\hline 2539.600 & 46 & 39364.45 & 0.002 & 75221.11 & $6-35856.62$ & 6 & H5p1-d & \\
\hline 2539.192 & 48 & 39370.79 & 0.002 & 76978.95 & $4-37608.12$ & 4 & F4p3-d & $2.651(8)$ \\
\hline 2563.775 & 80 & 39408.29 & 0.005 & 68186.11 & $6-28777.76$ & 6 & I6p3-I6d5 & $8.651(8)$ \\
\hline 2532.367 & 80 & 39476.88 & 0.006 & 61493.77 & $6-22016.80$ & 5 & $17 \mathrm{p} 3-17 \mathrm{~d} 5$ & $1.899(9)$ \\
\hline 2531.037 & 70 & 39497.62 & 0.004 & 66077.65 & $6-26579.97$ & 7 & I5p1-I6d3 & $3.016(8)$ \\
\hline 2529.431 & 70 & 39522.69 & 0.007 & 65934.64 & $5-26411.84$ & 6 & $15 p 1-I 6 s 1$ & $5.233(8)$ \\
\hline 2529.020 & 80 & 39529.12 & 0.010 & 68084.68 & $7-28555.40$ & 8 & I6p3-I6d5 & $2.073(9)$ \\
\hline 2527.243 & 46 & 39556.92 & 0.004 & 71459.21 & $4-31902.23$ & 4 & I5p3-I4s1 & $1.303(8)$ \\
\hline 2525.613 & 42 & 39582.44 & -0.004 & 6969.80 & $5-46552.18$ & 5 & $3 \mathrm{H}-? \mathrm{~d}$ & \\
\hline 2525.613 & 42 & 39582.44 & -0.001 & 71779.39 & $5-32196.96$ & 4 & I5p3-d & $1.647(8)$ \\
\hline 2523.662 & 41 & 39613.04 & 0.001 & 71459.21 & 4-31846.16 & 3 & I5p3-I4d5 & $3.826(8)$ \\
\hline 2521.293 & 40 & 39650.26 & 0.004 & 74944.47 & $5-35294.14$ & 4 & H5p1-F4s1 & $1.632(8)$ \\
\hline 2520.310 & 59 & 39665.73 & 0.005 & 66077.65 & $6-26411.84$ & 6 & I5p1-I6s1 & $2.358(8)$ \\
\hline 2515.486 & 48 & 39741.79 & 0.010 & 65934.64 & $5-26192.70$ & 5 & $15 \mathrm{p} 1-16 \mathrm{~d} 3$ & $2.104(8)$ \\
\hline 2509.926 & 80 & 39829.82 & -0.001 & 71531.27 & $7-31701.46$ & 8 & I5p3-I5d5 & $3.308(9)$ \\
\hline 2509.563 & 54 & 39835.57 & 0.002 & 71050.12 & $5-31214.52$ & 5 & F4p1-d & $1.934(8)$ \\
\hline 2509.245 & 46 & 39840.63 & 0.001 & 71055.16 & $4-31214.52$ & 5 & F4p1-d & $1.408(8)$ \\
\hline 2508.597 & 80 & 39850.92 & -0.001 & 61539.18 & $8-21688.27$ & 9 & I7p3-17d5 & $2.581(9)$ \\
\hline 2501.281 & 46 & 39967.48 & -0.003 & 6969.80 & $5-46937.23$ & 4 & $3 \mathrm{H}-$ & \\
\hline 2500.824 & 42 & 39974.77 & 0.006 & 66077.65 & $6-26102.79$ & 7 & I5p1-I6s1 & \\
\hline 2498.053 & 48 & 40019.11 & 0.002 & 74944.47 & $5-34925.33$ & 5 & H5p1-F4s1 & $2.937(8)$ \\
\hline 2494.910 & 46 & 40069.53 & -0.012 & 76724.94 & $6-36655.60$ & 5 & F4p3-d5 & \\
\hline 2493.756 & 70 & 40088.07 & 0.000 & 76724.94 & $6-36636.87$ & 7 & $\mathrm{~F} 4 \mathrm{p} 3-\mathrm{F} 4 \mathrm{~d} 5$ & 2.407 (9) \\
\hline 2492.050 & 70 & 40115.51 & 0.003 & 67986.38 & $5-27870.83$ & 5 & I6p3-16d5 & $3.383(8)$ \\
\hline 2489.936 & 43 & 40149.56 & -0.001 & 76480.35 & $4-36330.81$ & 5 & F3p1-d & \\
\hline 2489.606 & 59 & 40154.88 & -0.002 & 76724.94 & $6-36570.10$ & 6 & F4p3-I4d5 & $6.309(8)$ \\
\hline
\end{tabular}


Table I. Continued

\begin{tabular}{|c|c|c|c|c|c|c|c|c|}
\hline (1) & (2) & (3) & (4) & (5) & (6) (7) & (8) & (9) & (10) \\
\hline 2487.949 & 70 & 40181.63 & 0.001 & 76751.75 & $5-36570.10$ & 6 & F4p3-I4d5 & $1.225(9)$ \\
\hline 2485.111 & 70 & 40227.51 & 0.005 & 67699.20 & $8-27471.61$ & 9 & I6p3-I6d5 & $3.837(9)$ \\
\hline 2482.464 & 45 & 40270.40 & -0.001 & 76174.34 & $3-35903.96$ & 4 & F3p1-F4d & $4.977(8)$ \\
\hline 2480.645 & 46 & 40299.94 & -0.003 & 71050.12 & $5-30750.22$ & 4 & F4p1-d & $2.270(8)$ \\
\hline 2480.336 & 59 & 40304.95 & -0.001 & 71055.16 & $4-30750.22$ & 4 & F4p1-d & $4.996(8)$ \\
\hline 2479.704 & 70 & 40315.22 & 0.004 & 68186.11 & $6-27870.83$ & 5 & $16 \mathrm{p} 3-16 \mathrm{~d} 5$ & $7.258(8)$ \\
\hline 2476.818 & 43 & 40362.19 & 0.003 & 73395.34 & 3- 33033.10 & 4 & $14 p 3-d$ & $1.532(8)$ \\
\hline 2473.215 & 59 & 40420.98 & -0.002 & 76751.75 & $5-36330.81$ & 5 & F4p3-d & $5.757(8)$ \\
\hline 2467.542 & 43 & 40513.90 & 0.001 & 67986.38 & $5-27472.46$ & 6 & I6p3-I6d3 & \\
\hline 2464.610 & 70 & 40562.11 & 0.000 & 61032.44 & $9-20570.33$ & 10 & $17 \mathrm{p} 3-17 \mathrm{~d} 5$ & $4.358(9)$ \\
\hline 2463.740 & 59 & 40576.43 & -0.002 & 76480.35 & $4-35903.96$ & 4 & F3p1-F4d & $2.886(8))$ \\
\hline 2461.566 & 41 & 40612.26 & -0.003 & 68084.68 & $7-27472.46$ & 6 & $16 \mathrm{p} 3-16 \mathrm{~d} 3$ & \\
\hline 2459.391 & 42 & 40648.18 & -0.002 & 76978.95 & $4-36330.81$ & 5 & F4p3-d & $2.955(8)$ \\
\hline 2454.542 & 48 & 40728.47 & -0.001 & 73919.99 & $6-33191.53$ & 6 & I4p3-d & $4.692(8)$ \\
\hline 2454.502 & 48 & 40729.14 & -0.001 & 73920.66 & $5-33191.53$ & 6 & I4p3-d & $3.945(8)$ \\
\hline 2446.815 & 43 & 40857.08 & 0.001 & 0.00 & $6-40857.10$ & 5 & $3 \mathrm{H}-? \mathrm{~d}$ & $1.980(7)$ \\
\hline 2445.433 & 48 & 40880.17 & 0.002 & 76174.34 & 3- 35294.14 & 4 & F3p1-F4s1 & $4.770(8)$ \\
\hline 2439.441 & 41 & 40980.58 & -0.008 & 81837.54 & $4-40857.10$ & 5 & F3p3-?d & $2.729(8)$ \\
\hline 2436.148 & 48 & 41035.97 & -0.002 & 71050.12 & $5-30014.18$ & 6 & F4p1-I5s1 & \\
\hline 2434.680 & 54 & 41060.71 & -0.003 & 61539.18 & $8-102599.84$ & 8 & I7p3-177s & $2.624(9)$ \\
\hline 2432.590 & 59 & 41095.98 & 0.007 & 74129.19 & $4-33033.10$ & 4 & I4p3-d & $3.674(8)$ \\
\hline 2431.513 & 70 & 41114.19 & -0.001 & 61032.44 & 9- 19918.26 & 8 & $17 \mathrm{p} 3-17 \mathrm{~d} 3$ & $9.967(8)$ \\
\hline 2431.215 & 40 & 41119.21 & 0.001 & 67699.20 & $8-26579.97$ & 7 & I6p3-I6d3 & \\
\hline 2427.260 & 59 & 41186.22 & -0.001 & 76480.35 & $4-35294.14$ & 4 & $F 3 p 1-F 4 s 1$ & $4.833(8)$ \\
\hline 2426.542 & 70 & 41198.40 & -0.001 & 73395.34 & 3- 32196.96 & 4 & I4p3-d & $9.143(8)$ \\
\hline 2425.582 & 54 & 41214.71 & -0.004 & 61699.28 & $7-102913.92$ & 7 & I7p3-I77s & $2.708(9)$ \\
\hline 2423.878 & 59 & 41243.67 & -0.002 & 71050.12 & $5-29806.48$ & 5 & F4p1-I5s1 & $4.019(8)$ \\
\hline 2423.588 & 70 & 41248.62 & 0.004 & 71055.16 & $4-29806.48$ & 5 & F4p1-I5s1 & $1.216(9)$ \\
\hline 2422.471 & 80 & 41267.63 & -0.005 & 61493.77 & $6-20226.22$ & 7 & 17p3-17s1 & $5.228(9)$ \\
\hline 2420.242 & 70 & 41305.64 & -0.001 & 73919.99 & $6-32614.37$ & 5 & I4p3-d & $1.925(9)$ \\
\hline $2420.204 \mathrm{~T}$ & Bl & & & 73920.66 & 5- 32614.37 & 5 & I5p3-d & $1.764(9)$ \\
\hline 2419.818 & 80 & 41312.88 & 0.005 & 61539.18 & $8-20226.22$ & 7 & 17p3-17s1 & $3.471(9)$ \\
\hline 2418.359 & 70 & 41337.80 & -0.001 & 67986.38 & $5-26648.59$ & 4 & I6p3-I5d3 & $5.581(8)$ \\
\hline 2416.202 & 42 & 41374.70 & 0.002 & 61539.18 & $8-102913.92$ & 7 & I7p3-177s & $1.704(9)$ \\
\hline 2413.911 & 48 & 41413.95 & 0.006 & 77318.02 & $3-35903.96$ & 4 & F4p3-F4d & $3.051(8)$ \\
\hline 2413.552 & 48 & 41420.12 & 0.002 & 61493.77 & $6-102913.92$ & 7 & $17 \mathrm{p} 3-177 \mathrm{~s}$ & $2.543(9)$ \\
\hline 2412.993 & 70 & 41429.71 & 0.006 & 81837.54 & $4-40407.72$ & 3 & F3p3-d & \\
\hline 2411.977 & 70 & 41447.16 & 0.000 & 80712.97 & $6-39265.81$ & 5 & H5p3- & \\
\hline 2411.369 & 70 & 41457.61 & 0.000 & 76751.75 & $5-35294.14$ & 4 & $F 4 p 3-F 4 s 1$ & $2.158(9)$ \\
\hline 2411.031 & 41 & 41463.43 & 0.009 & 71459.21 & $4-29995.62$ & 5 & $15 p 3-15 d 3$ & \\
\hline 2410.630 & 45 & 41470.32 & 0.005 & 5081.77 & $4-46552.18$ & 5 & $3 F-? d$ & \\
\hline 2410.472 & 75 & 41473.04 & 0.001 & 61699.28 & $7-20226.22$ & 7 & $17 \mathrm{p} 3-17 \mathrm{~s} 1$ & $5.415(9)$ \\
\hline 2409.308 & 70 & 41493.08 & 0.002 & 73395.34 & $3-31902.23$ & 4 & I4p3-I4s1 & $2.578(9)$ \\
\hline 2409.125 & 41 & 41496.23 & 0.004 & 71779.39 & $5-30283.09$ & 6 & I5p3-I5d3 & \\
\hline 2408.746 & 59 & 41502.76 & -0.008 & 71785.72 & $6-30283.09$ & 6 & $15 p 3-15 d 3$ & $4.827(8)$ \\
\hline 2407.911 & 70 & 41517.14 & -0.003 & 71531.27 & $7-30014.18$ & 6 & I5p3-15s1 & $6.839(9)$ \\
\hline 2405.717 & 70 & 41555.01 & 0.001 & 76480.35 & $4-34925.33$ & 5 & F3p1-F4s1 & $2.314(8)$ \\
\hline 2404.998 & 48 & 41567.42 & -0.001 & 61032.44 & $9-102599.84$ & 8 & $17 \mathrm{p} 3-177 \mathrm{~s}$ & $4.965(9)$ \\
\hline 2404.586 & 70 & 41574.56 & -0.001 & 67986.38 & $5-26411.85$ & 6 & I6p3-I6s1 & $4.897(9)$ \\
\hline 2403.763 & 70 & 41588.78 & -0.002 & 71055.16 & $4-29466.42$ & 3 & F4p1-Id & $2.024(8)$ \\
\hline 2403.320 & 75 & 41596.45 & -0.002 & 67699.20 & $8-26102.79$ & 7 & 16p3-16s1 & $8.960(9)$ \\
\hline 2402.760 & 59 & 41606.14 & 0.000 & 68186.11 & $6-26579.97$ & 7 & I6p3-I6d3 & $3.698(8)$ \\
\hline 2400.071 & 70 & 41652.75 & -0.001 & 71459.21 & $4-29806.48$ & 5 & I5p3-I5s1 & $3.287(9)$ \\
\hline 2399.018 & 59 & 41671.03 & 0.003 & 81837.54 & $4-40166.45$ & 4 & F3p3-F3s1 & \\
\hline 2398.913 & 59 & 41672.87 & -0.002 & 68084.68 & $7-26411.84$ & 6 & I6p3-I6s1 & $2.912(9)$ \\
\hline 2398.226 & 70 & 41684.79 & 0.001 & 76978.95 & $4-35294.14$ & 4 & F4p3-F4s1 & $3.064(9)$ \\
\hline 2396.404 & 70 & 41716.48 & 0.000 & 61032.44 & 9- 19315.96 & 8 & I7p3-I7s1 & $9.268(9)$ \\
\hline 2395.986 & 46 & 41723.76 & -0.003 & 73920.66 & $5-32196.96$ & 4 & I4p3-d & \\
\hline 2395.813 & 70 & 41726.78 & 0.001 & 73919.99 & $6-32193.19$ & 5 & I4p3-I4s1 & $4.732(9)$ \\
\hline 2393.609 & 70 & 41765.20 & 0.001 & 71779.39 & $5-30014.18$ & 6 & I5p3-I5s1 & $1.491(9)$ \\
\hline 2393.258 & 70 & 41771.32 & 0.013 & 71785.72 & $6-30014.18$ & 6 & I5p3-I5s1 & $3.986(9)$ \\
\hline 2393.083 & 70 & 41774.37 & -0.006 & 68186.11 & $6-26411.84$ & 6 & I6p3-I6s1 & 4.909 (9) \\
\hline 2392.921 & 40 & 41777.20 & 0.009 & 6969.80 & $5-48747.15$ & 5 & $3 \mathrm{H}-\mathrm{dd}$ & \\
\hline 2392.708 & 45 & 41780.92 & 0.006 & 61699.28 & $7-19918.26$ & 8 & I7p3-I7d3 & \\
\hline 2392.546 & 46 & 41783.76 & 0.001 & 71779.39 & $5-29995.62$ & 5 & I5p3-I5d3 & $6.385(8)$ \\
\hline 2391.970 & 70 & 41793.81 & -0.007 & 67986.38 & $5-26192.70$ & 5 & I6p3-I6d3 & $3.063(8)$ \\
\hline 2391.637 & 70 & 41799.63 & -0.001 & 76724.94 & $6-34925.33$ & 5 & $\mathrm{~F} 4 \mathrm{p} 3-\mathrm{F} 4 \mathrm{~s} 1$ & $6.584(9)$ \\
\hline 2390.109 & 70 & 41826.34 & 0.005 & 76751.75 & $5-34925.33$ & 5 & $F 4 p 3-F 4 s 1$ & $2.421(9)$ \\
\hline 2388.191 & 59 & 41859.95 & 0.000 & 80712.97 & $6-38853.02$ & 6 & H5p3- & \\
\hline 2385.254 & 43 & 41911.48 & -0.006 & 74944.47 & $5-33033.10$ & 4 & H5p1-d & \\
\hline 2384.755 & 38 & 41920.25 & 0.002 & 71531.27 & $7-29610.99$ & 7 & $15 \mathrm{p} 3-16 \mathrm{~d} 5$ & \\
\hline
\end{tabular}


Table I. Continued

\begin{tabular}{|c|c|c|c|c|c|c|c|c|}
\hline (1) & (2) & (3) & (4) & (5) & (6) (7) & (8) & (9) & (10) \\
\hline 2384.755 . & 38 & 41920.25 & 0.007 & 68332.21 & $5-26411.84$ & 6 & I4p1-I6s1 & $1.526(8)$ \\
\hline 2384.114 & 59 & 41931.51 & -0.003 & 80712.97 & $6-38781.51$ & 6 & H5p3- & \\
\hline 2384.072 & 59 & 41932.25 & -0.001 & 74129.19 & $4-32196.96$ & 4 & I4p3-d & $3.891(8)$ \\
\hline 2383.855 & 70 & 41936.07 & -0.004 & 74129.19 & $4-32193.19$ & 5 & $14 \mathrm{p} 3-I 4 s 1$ & $1.694(9)$ \\
\hline 2381.756 & 59 & 41973.02 & -0.006 & 71779.39 & 5- 29806.48 & 5 & I5p3-I5s1 & 3.257 (9) \\
\hline 2381.402 & 59 & 41979.26 & -0.001 & 71785.72 & $6-29806.48$ & 5 & $15 \mathrm{p} 3-\mathrm{I} 5 \mathrm{~s} 1$ & $2.150(9)$ \\
\hline 2381.250 & 59 & 41981.94 & -0.003 & 68084.68 & $7-26102.79$ & 7 & I6p3-I6s1 & $4.994(9)$ \\
\hline 2380.804 & 48 & 41989.81 & 0.000 & 71459.21 & $4-29469.40$ & 4 & I5p3-Id & $3.813(8)$ \\
\hline 2380.628 & 46 & 41992.92 & -0.007 & 71459.21 & $4-29466.42$ & 3 & I5p3-Id & $3.374(8)$ \\
\hline 2379.179 & 59 & 42018.49 & -0.003 & 73920.66 & $5-31902.23$ & 4 & $\mathrm{I} 4 \mathrm{p}^{3}-\mathrm{I} 4 \mathrm{~s} 1$ & $1.637(9)$ \\
\hline 2378.872 & 59 & 42023.91 & -0.002 & 77318.02 & 3- 35294.14 & 4 & $\mathrm{~F} 4 \mathrm{p} 3-\mathrm{F} 4 \mathrm{~s} 1$ & $2.589(9)$ \\
\hline 2377.186 & 59 & 42053.72 & -0.006 & 76978.95 & $4-34925.33$ & 5 & $\mathrm{~F} 4 \mathrm{p} 3-\mathrm{F} 4 \mathrm{~s} 1$ & $1.349(9)$ \\
\hline 2377.077 & 59 & 42055.64 & -0.001 & 61032.44 & 9- 18976.82 & 9 & $17 \mathrm{p} 3-17 \mathrm{~d} 3$ & $6.033(8)$ \\
\hline 2375.511 & 59 & 42083.36 & -0.002 & 68186.11 & $6-26102.79$ & 7 & I6p3-I6s1 & $1.373(9)$ \\
\hline 2370.336 & 59 & 42175.23 & -0.028 & 71785.72 & $6-29610.99$ & 7 & I5p3-I6d5 & $2.192(8)$ \\
\hline 2367.988 & 54 & 42217.05 & -0.005 & 67699.20 & $8-25482.23$ & 8 & $\mathrm{I} 6 \mathrm{p} 3-\mathrm{I} 6 \mathrm{~d} 3$ & \\
\hline 2367.641 & 59 & 42223.24 & -0.001 & 61539.18 & $8-19315.96$ & 8 & $17 \mathrm{p} 3-17 \mathrm{~s} 1$ & $5.926(9)$ \\
\hline 2367.433 & 59 & 42226.95 & 0.001 & 74129.19 & $4-31902.23$ & 4 & $\mathrm{I} 4 \mathrm{p} 3-\mathrm{I} 4 \mathrm{~s} 1$ & $2.941(9)$ \\
\hline 2359.332 & 59 & 42371.93 & 0.000 & 62598.14 & $6-20226.22$ & 7 & I6p1-I7s1 & $1.497(9)$ \\
\hline 2358.793 & 59 & 42381.60 & 0.002 & 62607.86 & $7-20226.22$ & 7 & I6p1-I7s 1 & $6.505(8)$ \\
\hline 2358.699 & 59 & 42383.30 & 0.001 & 61699.28 & $7-19315.96$ & 8 & $17 \mathrm{p} 3-\mathrm{I} 7 \mathrm{~s} 1$ & $1.529(9)$ \\
\hline 2348.778 & 45 & 42562.31 & 0.003 & 61539.18 & $8-18976.82$ & 9 & $17 \mathrm{p} 3-17 \mathrm{~d} 3$ & $1.675(8)$ \\
\hline 2348.256 & 45 & 42571.75 & -0.001 & 81837.54 & $4-39265.81$ & 5 & F3p3- & \\
\hline 2344.217 & 41 & 42645.10 & 0.001 & 73395.34 & $3-30750.22$ & 4 & $14 \mathrm{p} 3-\mathrm{d}$ & $2.199(8)$ \\
\hline 2340.495 & 46 & 42712.92 & -0.005 & 71531.27 & $7-28818.44$ & 7 & I5p3-I5d3 & \\
\hline 2338.394 & 59 & 42751.28 & 0.000 & 74944.47 & 5- 32193.19 & 5 & H5p1-I4s1 & $3.279(8)$ \\
\hline 2338.270 & 41 & 42753.57 & -0.002 & 71531.27 & $7-28777.74$ & 6 & I5p3-I6d5 & \\
\hline 2334.417 & 47 & 42824.12 & 0.003 & 73919.99 & $6-31095.82$ & 6 & $14 \mathrm{p3}-14 \mathrm{~d} 3$ & $1.734(8)$ \\
\hline 2334.376 & 48 & 42824.86 & -0.001 & 73920.66 & $5-31095.82$ & 6 & I4p3-14d3 & $2.485(8)$ \\
\hline 2329.566 & 48 & 42913.29 & -0.003 & 81837.54 & 4- 38924.30 & 3 & F3p3- & \\
\hline 2329.483 & 59 & 42914.81 & -0.007 & 74129.19 & $4-31214.52$ & 5 & I4p3-d & $3.086(8)$ \\
\hline 2326.635 & 48 & 42967.33 & -0.003 & 71785.72 & $6-28818.44$ & 7 & $15 \mathrm{p} 3-15 \mathrm{~d} 3$ & $2.355(8)$ \\
\hline 2325.480 & 38 & 42988.68 & 0.001 & 76174.34 & 3- 33185.64 & 3 & F3p1-F4d3 & \\
\hline 2324.775 & 46 & 43001.71 & -0.003 & 71779.39 & 5- 28777.74 & 6 & I5p3-I6d5 & $1.572(8)$ \\
\hline 2324.435 & 41 & 43008.01 & -0.002 & 71785.72 & $6-28777.74$ & 6 & I5p3-I6d5 & \\
\hline 2323.359 & 43 & 43027.91 & 0.001 & 75221.11 & $6-32193.19$ & 5 & H5p1-I4s1 & \\
\hline 2322.589 & 59 & 43042.19 & 0.003 & 74944.47 & $5-31902.23$ & 4 & H5p1-I4s1 & $2.391(8)$ \\
\hline 2309.194 & 59 & 43291.84 & 0.003 & 62607.86 & $7-19315.96$ & 8 & $16 \mathrm{p} 1-\mathrm{I} 7 \mathrm{~s} 1$ & $2.306(8)$ \\
\hline 2296.380 & 70 & 43533.39 & 0.001 & 76724.94 & $6-33191.53$ & 6 & F4p3-d & $4.423(8)$ \\
\hline 2290.894 & 40 & 43637.62 & -0.003 & 73920.66 & $5-30283.09$ & 6 & $\mathrm{I} 4 \mathrm{p} 3-\mathrm{I} 5 \mathrm{~d} 3$ & \\
\hline 2289.438 & 45 & 43665.37 & 0.000 & 5081.77 & $4-48747.15$ & 5 & $3 F-? d$ & \\
\hline 2277.654 & 65 & 43891.26 & 0.001 & 61539.18 & $8-17647.90$ & 7 & I7p3-I7d3 & $7.468(8)$ \\
\hline 2276.433 & 59 & 43914.80 & 0.005 & 71785.72 & 6- 27870.83 & 5 & $15 \mathrm{p} 3-16 \mathrm{~d} 5$ & $1.858(8)$ \\
\hline 2275.904 & 48 & 43925.02 & 0.001 & 73920.66 & $5-29995.62$ & 5 & $\mathrm{I} 4 \mathrm{p} 3-\mathrm{I} 5 \mathrm{~d} 3$ & $1.782(8)$ \\
\hline 2275.704 & 46 & 43928.88 & 0.002 & 73395.34 & $3-29466.42$ & 3 & I4p3-Id & $1.587(8)$ \\
\hline 2269.376 & 59 & 44051.35 & 0.001 & 61699.28 & $7-17647.90$ & 7 & $17 \mathrm{p} 3-17 \mathrm{~d} 3$ & $2.149(8)$ \\
\hline 2268.994 & 43 & 44058.78 & 0.001 & 71531.27 & $7-27472.46$ & 6 & $15 \mathrm{p3}-16 \mathrm{~d} 3$ & \\
\hline 2265.210 & 45 & 44132.37 & 0.000 & 77318.02 & 3- 33185.64 & 3 & $\mathrm{~F} 4 \mathrm{p} 3-\mathrm{F} 4 \mathrm{~d} 3$ & $1.763(8)$ \\
\hline 2262.040 & 47 & 44194.20 & 0.003 & 74944.47 & $5-30750.22$ & 4 & H5p1-d & \\
\hline 2255.967 & 46 & 44313.17 & 0.005 & 71785.72 & $6-27472.46$ & 6 & $15 \mathrm{p} 3-16 \mathrm{~d} 3$ & \\
\hline 2252.463 & 43 & 44382.09 & 0.004 & 80712.97 & $6-36330.81$ & 5 & H5p3-d & \\
\hline 2248.962 & 45 & 44451.19 & 0.004 & 73920.66 & 5- 29469.40 & 4 & I4p3-Id & \\
\hline 2245.615 & 46 & 44517.43 & 0.003 & 61493.77 & $6-16976.28$ & 6 & $\mathrm{I} 7 \mathrm{p} 3-17 \mathrm{~d} 3$ & \\
\hline 2244.898 & 45 & 44531.65 & 0.005 & 76724.94 & $6-32193.19$ & 5 & F4p3-I4s1 & \\
\hline 2235.292 & 59 & 44722.99 & 0.000 & 61699.28 & $7-16976.28$ & 6 & $17 \mathrm{p} 3-17 \mathrm{~d} 3$ & $3.178(8)$ \\
\hline 2232.360 & 48 & 44781.73 & 0.003 & 68084.68 & $7-23302.89$ & 7 & I6p3-I7d5 & \\
\hline 2198.158 & 41 & 45478.43 & 0.006 & 68084.68 & $7-22606.12$ & 6 & I6p3-I7d5 & $3.117(8)$ \\
\hline 2193.262 & 39 & 45579.95 & 0.002 & 68186.11 & $6-22606.12$ & 6 & $16 \mathrm{p} 3-17 \mathrm{~d} 5$ & \\
\hline 2190.780 & 39 & 45631.58 & 0.000 & 62607.86 & $7-16976.28$ & 6 & I6p1-I7d3 & \\
\hline 2165.267 & 41 & 46169.18 & 0.006 & 68186.11 & $6-22016.80$ & 5 & I6p3-I7d5 & \\
\hline 2132.044 & 42 & 46888.54 & -0.001 & 56025.40 & $8-102913.92$ & 7 & I7p1-I77s & $3.933(9)$ \\
\hline 2124.614 & 45 & 47052.50 & 0.002 & 55547.30 & $7-102599.84$ & 8 & $17 \mathrm{pl}-\mathrm{I} 77 \mathrm{~s}$ & $4.566(9)$ \\
\hline
\end{tabular}

Notes: Lines noted NS in the second column (Intensity) are taken from Ref. [2].

2 Possibly blend with an Er II line.

- Wavelength from Ref. [2], the deviation with Ref. [9] is too large.

${ }^{b}$ Revised wavelength from $H$. Crosswhite.

- Theoretical wavelength, masked by a strong Er II line.

d Given as Er II in Ref. [9] and absent in Ref. [2].

- Theoretical wavelength, absent in Ref. [2] and [9], but strong in HR 465 (Ref. [8]).

' Theoretical wavelength, far from 3473.870 (Ref. [9])

B Theoretical wavelength, far from 3469.127 (Ref. [9]) 
Table II. Energy levels of Er III with main components of their eigenfunctions and gth Landé factors. Leading components smaller than $33 \%$ are omitted.

\begin{tabular}{|c|c|c|c|c|c|c|c|c|}
\hline \multicolumn{9}{|c|}{ Configuration $4 f^{12}$} \\
\hline$L S$-Term & $J$ & $\begin{array}{l}\text { Energy } \\
\left(\mathrm{cm}^{-1}\right)\end{array}$ & $\begin{array}{l}\text { 1st Comp. } \\
\%\end{array}$ & 2nd & $\%$ & $g_{\text {th }}$ & Note & $\begin{array}{l}\text { Label in } \\
\text { Table I }\end{array}$ \\
\hline${ }^{3} \mathrm{H}$ & 6 & 0.00 & 99.2 & ${ }^{1} \mathrm{I}$ & 0.8 & 1.165 & a & $3 \mathrm{H}$ \\
\hline${ }^{3} \mathrm{H}$ & 5 & 6969.80 & 100.0 & & 0.0 & 1.033 & a & $3 \mathrm{H}$ \\
\hline${ }^{3} \mathrm{H}$ & 4 & 10785.51 & 61.5 & ${ }^{3} \mathrm{G}$ & 27.0 & 1.139 & b & $3 \mathrm{H}$ \\
\hline${ }^{3} \mathrm{~F}$ & 4 & 5081.77 & 62.7 & ${ }^{1} \mathrm{G}$ & 28.7 & 0.945 & a & $3 \mathrm{~F}$ \\
\hline${ }^{3} \mathrm{~F}$ & 3 & 12472.55 & 100.0 & & 0.0 & 1.083 & $\mathbf{N}$ & $3 F$ \\
\hline${ }^{3} \mathrm{~F}$ & 2 & 13219.80 & 79.9 & ${ }^{1} \mathrm{D}$ & 18.6 & 0.732 & $\mathbf{N}$ & $3 F$ \\
\hline${ }^{1} \mathrm{G}$ & 4 & 18383.59 & 59.8 & ${ }^{3} \mathrm{H}$ & 29.9 & 0.966 & $\mathbf{N}$ & $1 \mathrm{G}$ \\
\hline
\end{tabular}

Configuration $4 f^{11} 6 \mathrm{p}$

\begin{tabular}{|c|c|c|c|c|c|c|c|}
\hline Multiplet & $J$ & $\begin{array}{l}\text { Energy } \\
\left(\mathrm{cm}^{-1}\right)\end{array}$ & $\begin{array}{l}\text { 1st Comp. } \\
\%\end{array}$ & & $g$ th & Note & \\
\hline \multirow[t]{2}{*}{${ }^{4} I_{15 / 2} P_{1 / 2}$} & 7 & 55547.30 & 96 & & 1.243 & a & $17 \mathrm{pl}$ \\
\hline & 8 & 56025.40 & 98 & & 1.164 & a & I7pI \\
\hline \multirow[t]{4}{*}{${ }^{4} I_{15 / 2} p_{3 / 2}$} & 9 & 61032.44 & 98 & & 1.219 & a & $17 \mathrm{p} 3$ \\
\hline & 6 & 61493.77 & 72 & & 1.170 & a & $17 p 3$ \\
\hline & 8 & 61539.18 & 98 & & 1.207 & a & $17 \mathrm{p} 3$ \\
\hline & 7 & 61699.28 & 84 & & 1.167 & $\mathbf{a}$ & I7p3 \\
\hline \multirow{2}{*}{${ }^{4} I_{13 / 2} P_{1 / 2}$} & 6 & 62598.14 & 74 & & 1.146 & $\mathbf{a}$ & I6p1 \\
\hline & 7 & 62607.86 & 86 & & 1.086 & a & I6p1 1 \\
\hline \multirow{2}{*}{${ }^{4} I_{11 / 2} p_{1 / 2}$} & 5 & 65934.64 & 79 & & 1.026 & a & $15 \mathrm{p} 1$ \\
\hline & 6 & 66077.65 & 82 & & 0.964 & $\mathbf{a}$ & $15 \mathrm{p} 1$ \\
\hline \multirow[t]{4}{*}{${ }^{4} I_{13 / 2} P_{3 / 2}$} & 8 & 67699.20 & 99 & & 1.148 & c & I6p3 \\
\hline & 5 & 67986.38 & 90 & & 1.047 & $\mathrm{a}$ & I6p3 \\
\hline & 7 & 68084.68 & 99 & & 1.130 & a & I6p3 \\
\hline & 6 & 68186.11 & 95 & & $\cdot 1.088$ & a & I6p3 \\
\hline \multirow{2}{*}{${ }^{4} I_{9 / 2} p_{1 / 2}$} & 4 & 68234.37 & 50 & & 0.937 & $\mathbf{N}$ & I4p1 \\
\hline & 5 & 68332.21 & 54 & & 0.876 & $\mathbf{N}$ & $14 \mathrm{p} 1$ \\
\hline \multirow{2}{*}{${ }^{4} F_{9 / 2} p_{1 / 2}$} & 5 & 71050.12 & 54 & & 1.100 & $\mathbf{N}$ & F4p1 \\
\hline & 4 & 71055.16 & 50 & & 1.128 & $\mathbf{N}$ & F4p1 \\
\hline \multirow{4}{*}{${ }^{4} I_{11 / 2} P_{3 / 2}$} & 4 & 71459.21 & 61 & & 0.932 & $\mathbf{N}$ & $15 \mathrm{p} 3$ \\
\hline & 7 & 71531.27 & 83 & & 1.061 & $\mathbf{N}$ & $15 \mathrm{p} 3$ \\
\hline & 5 & 71779.39 & 72 & & 0.983 & a & $15 \mathrm{p} 3$ \\
\hline & 6 & 71785.72 & 81 & & 1.036 & a & I5p3 \\
\hline \multirow{4}{*}{${ }^{4} I_{9 / 2} p_{3 / 2}$} & 3 & 73395.34 & 66 & & 0.651 & $\mathbf{N}$ & I4p3 \\
\hline & 6 & 73919.99 & 52 & & 1.011 & $\mathbf{N}$ & $14 \mathrm{p} 3$ \\
\hline & 5 & 73920.66 & 38 & & 1.032 & $\mathbf{N}$ & I4p3 \\
\hline & 4 & 74129.19 & 56 & & 0.867 & $\mathbf{N}$ & I4p3 \\
\hline \multirow[t]{2}{*}{${ }^{2} \mathrm{H}_{11 / 2} \mathrm{p}_{1 / 2}$} & 5 & 74944.47 & 40 & & 1.122 & $\mathbf{N}$ & H5p1 \\
\hline & 6 & 75221.11 & 52 & & 1.091 & $\mathbf{N}$ & H5p1 \\
\hline \multirow[t]{2}{*}{${ }^{4} F_{7 / 2} P_{1 / 2}$} & 3 & 76174.34 & 82 & & 1.239 & $\mathbf{N}$ & F3p1 \\
\hline & 4 & 76480.35 & 85 & & 1.161 & $\mathbf{N}$ & F3p1 \\
\hline \multirow{4}{*}{${ }^{4} \mathrm{~F}_{9 / 2} \mathrm{P}_{3 / 2}$} & 6 & 76724.94 & 61 & & 1.183 & $\mathbf{N}$ & F4p3 \\
\hline & 5 & 76751.75 & 53 & & 1.143 & $\mathbf{N}$ & F4p3 \\
\hline & 4 & 76978.95 & 60 & & 1.153 & $\mathbf{N}$ & F4p3 \\
\hline & 3 & 77318.02 & 56 & & 1.178 & $\mathbf{N}$ & F4p3 \\
\hline${ }^{2} \mathrm{H}_{11 / 2} \mathrm{p}_{3 / 2}$ & 6 & 80712.97 & 53 & & 1.153 & $\mathbf{N}$ & H5p3 \\
\hline${ }^{4} \mathrm{~F}_{7 / 2} \mathrm{p}_{3 / 2}$ & 4 & 81837.54 & 54 & & 1.178 & $\mathbf{N}$ & F3p3 \\
\hline \multicolumn{8}{|c|}{ Configuration $4 f^{11} 5 d+4 f^{11} 6 s$} \\
\hline Multiplet & $J$ & $\begin{array}{l}\text { Energy } \\
\left(\mathrm{cm}^{-1}\right)\end{array}$ & $\begin{array}{l}\text { First } \\
\text { Comp. \% }\end{array}$ & $\begin{array}{l}4 f^{11} 6 s \\
\%\end{array}$ & $g$ th & Note & $\begin{array}{l}\text { Label in } \\
\text { Table I }\end{array}$ \\
\hline \multirow[t]{4}{*}{${ }^{4} I_{15 / 2} d_{3 / 2}$} & 6 & 16976.28 & 91 & 0.0 & 1.301 & a & $17 \mathrm{~d} 3$ \\
\hline & 7 & 17647.90 & 78 & 0.2 & 1.262 & a & $17 \mathrm{~d} 3$ \\
\hline & 9 & 18976.82 & 95 & 0.0 & 1.137 & a & $17 \mathrm{~d} 3$ \\
\hline & 8 & 19918.26 & 92 & 0.1 & 1.179 & a & $17 \mathrm{~d} 3$ \\
\hline \multirow[t]{2}{*}{${ }^{4} I_{15 / 2} s_{1 / 2}$} & 8 & 19315.96 & 97 & 99.9 & 1.246 & a & I7s1 \\
\hline & 7 & 20226.22 & 96 & 99.3 & 1.148 & a & $17 \mathrm{~s} 1$ \\
\hline \multirow[t]{6}{*}{${ }^{4} I_{15 / 2} d_{3 / 2}$} & 10 & 20470.33 & 96 & 0.0 & 1.197 & a & $17 \mathrm{~d} 5$ \\
\hline & 9 & 21688.27 & 96 & 0.0 & 1.191 & $\mathbf{a}$ & I7d 5 \\
\hline & 5 & 22016.80 & 51 & 0.0 & 1.211 & a & $\mathrm{I} 7 \mathrm{~d} 5$ \\
\hline & 6 & 22606.24 & 52 & 0.1 & 1.205 & $\mathbf{a}$ & I7d 5 \\
\hline & 8 & 22951.53 & 90 & 0.1 & 1.178 & a & I7d5 \\
\hline & 7 & 23302.89 & 65 & 0.3 & 1.145 & $\mathbf{a}$ & I7d 5 \\
\hline${ }^{4} I_{13 / 2} d_{3 / 2}$ & 8 & 25482.23 & 91 & 0.0 & 1.053 & c & $16 \mathrm{~d} 3$ \\
\hline
\end{tabular}


Table II. Continued

\begin{tabular}{|c|c|c|c|c|c|c|c|}
\hline \multicolumn{8}{|c|}{ Configuration $4 f^{11} 5 d+4 f^{11} 6 s$} \\
\hline Multiplet & $J$ & $\begin{array}{l}\text { Energy } \\
\left(\mathrm{cm}^{-1}\right)\end{array}$ & $\begin{array}{l}\text { First } \\
\text { Comp. \% }\end{array}$ & $\begin{array}{l}4 f^{11} 6 s \\
\%\end{array}$ & gth & Note & $\begin{array}{l}\text { Label in } \\
\text { Table I }\end{array}$ \\
\hline${ }^{4} I_{13 / 2} s_{1 / 2}$ & 7 & 26102.79 & 97 & 97.6 & 1.167 & a & $16 \mathrm{~d} 3$ \\
\hline${ }^{4} I_{13 / 2} d_{3 / 2}$ & 5 & 26192.70 & 44 & 0.3 & 1.140 & a & $\mathrm{I} 6 \mathrm{~d} 3$ \\
\hline${ }^{4} I_{13 / 2} s_{1 / 2}$ & 6 & 26411.84 & 97 & 99.3 & 1.049 & a & I6s1 \\
\hline${ }^{4} I_{13 / 2} d_{3 / 2}$ & 7 & 26579.97 & 74 & 2.2 & 1.055 & $\mathrm{a}$ & $16 \mathrm{~d} 3$ \\
\hline${ }^{4} I_{11 / 2} d_{3 / 2}$ & 4 & 26648.59 & 45 & 0.0 & 1.071 & $\mathbf{N}$ & $15 \mathrm{~d} 3$ \\
\hline${ }^{4} I_{13 / 2} d_{5 / 2}$ & 9 & 27471.61 & 98 & 0.0 & 1.131 & $\mathbf{N}$ & $16 \mathrm{~d} 5$ \\
\hline${ }^{4} I_{13 / 2} d_{3 / 2}$ & 6 & 27472.46 & 56 & 0.5 & 1.077 & a & $\mathrm{I} 6 \mathrm{~d} 3$ \\
\hline${ }^{4} I_{13 / 2} d_{5 / 2}$ & 5 & 27870.83 & 50 & 0.3 & 1.165 & a & I6d5 \\
\hline${ }^{4} I_{13 / 2} d_{5 / 2}$ & 8 & 28555.40 & 97 & 0.0 & 1.130 & c & I6d5 \\
\hline${ }^{4} I_{13 / 2} d_{5 / 2}$ & 6 & 28777.74 & 60 & 3.2 & 1.080 & a & $\mathrm{I} 6 \mathrm{~d} 5$ \\
\hline${ }^{4} I_{11 / 2} d_{3 / 2}$ & 7 & 28818.44 & 51 & 0.1 & 1.020 & a & $\mathrm{I} 5 \mathrm{~d} 3$ \\
\hline${ }^{4} I_{11 / 2} d_{3 / 2}$ & 3 & 29466.42 & 34 & 0.0 & 0.829 & $N$ & Id \\
\hline $4 f^{11} 5 d$ & 4 & 29469.40 & & 0.1 & 1.051 & $\mathbf{N}$ & Id \\
\hline${ }^{4} I_{13 / 2} d_{5 / 2}$ & 7 & 29610.99 & 66 & 0.1 & 1.068 & d & I6d5 \\
\hline${ }^{4} I_{11 / 2} s_{1 / 2}$ & 5 & 29806.48 & 71 & 82.9 & 0.920 & a & I5s 1 \\
\hline${ }^{4} I_{11 / 2} d_{3 / 2}$ & 5 & 29995.62 & 36 & 16.8 & 0.977 & $\mathrm{~N}$ & $15 \mathrm{~d} 3$ \\
\hline${ }^{4} I_{11 / 2} s_{1 / 2}$ & 6 & 30014.18 & 75 & 94.7 & 1.068 & e & I5s 1 \\
\hline${ }^{4} \mathrm{I}_{11 / 2} \mathrm{~d}_{3 / 2}$ & 6 & 30283.09 & 39 & 1.1 & 0.949 & e & $\mathrm{I} 5 \mathrm{~d} 3$ \\
\hline${ }^{4} \mathrm{~F}_{9 / 2} \mathrm{~d}_{3 / 2}$ & 4 & 30750.22 & 37 & 0.2 & 1.144 & $\mathbf{N}$ & d \\
\hline${ }^{4} I_{9 / 2} d_{3 / 2}$ & 6 & 31095.82 & 50 & 0.9 & 0.870 & e & $\mathrm{I} 4 \mathrm{~d} 3$ \\
\hline $4 \mathrm{f}^{11} 5 \mathrm{~d}$ & 5 & 31214.52 & & 1.3 & 1.047 & $\mathbf{N}$ & $d$ \\
\hline${ }^{4} I_{11 / 2} d_{5 / 2}$ & 8 & 31701.46 & 81 & 0.0 & 1.052 & $\mathbf{N}$ & I5d5 \\
\hline${ }^{4} \mathrm{I}_{9 / 2} \mathrm{~d}_{5 / 2}$ & 3 & 31846.16 & 45 & 0.0 & 0.808 & $\mathbf{N}$ & $\mathrm{I} 4 \mathrm{~d} 5$ \\
\hline${ }^{4} I_{9 / 2} s_{1 / 2}$ & 4 & 31902.23 & 44 & 82.2 & 0.824 & $\mathbf{N}$ & I $4 \mathrm{~s} 1$ \\
\hline${ }^{4} I_{9 / 2} s_{1 / 2}$ & 5 & 32193.19 & & 55.3 & 1.037 & $\mathbf{N}$ & I 4 s 1 \\
\hline $4 f^{11} 5 d$ & 4 & 32196.96 & & 16.5 & 0.940 & $\mathrm{~N}$ & $d$ \\
\hline${ }^{4} I_{11 / 2} d_{s / 2}$ & 7 & 32559.55 & 67 & 0.1 & 1.067 & $\mathbf{N}$ & I5d5 \\
\hline $4 f^{11} 5 d$ & 5 & 32614.37 & & 41.9 & 1.010 & $\mathrm{~N}$ & d \\
\hline $4 f^{11} 5 d$ & 4 & 33033.10 & & 1.2 & 0.947 & $\mathbf{N}$ & d \\
\hline${ }^{4} F_{9 / 2} d_{3 / 2}$ & 3 & 33185.64 & 48 & 0.1 & 1.209 & $\mathbf{N}$ & $\mathrm{F} 4 \mathrm{~d} 3$ \\
\hline $4 \mathrm{f}^{11} 5 \mathrm{~d}$ & 6 & 33191.53 & & 0.0 & 1.150 & $\mathbf{N}$ & d \\
\hline $4 f^{11} 5 d$ & 5 & 33704.29 & & 1.5 & 0.976 & $\mathrm{~N}$ & $\mathrm{~d}$ \\
\hline${ }^{4} I_{11 / 2} d_{5 / 2}$ & 6 & 33875.19 & 57 & 0.3 & 1.055 & e & I5d5 \\
\hline${ }^{4} \mathrm{~F}_{9 / 2} \mathrm{~s}_{1 / 2}$ & 5 & 34925.33 & 56 & 99.0 & 1.196 & $\mathbf{N}$ & $\mathrm{F} 4 \mathrm{~s} 1$ \\
\hline${ }^{4} F_{9 / 2} s_{1 / 2}$ & 4 & 35294.14 & 60 & 91.6 & 1.103 & $\mathbf{N}$ & $\mathrm{F} 4 \mathrm{~s} 1$ \\
\hline $4 \mathrm{f}^{11} 5 \mathrm{~d}$ & 6 & 35856.62 & & 0.2 & 1.080 & $\mathrm{~N}$ & $d$ \\
\hline${ }^{4} F_{9 / 2} d$ & 4 & 35903.96 & & 6.7 & 1.161 & $\mathbf{N}$ & F4d \\
\hline $4 f^{115} d$ & 3 & 36167.30 & & 0.4 & 0.928 & $\mathbf{N}$ & d \\
\hline $4 f^{11} 5 d$ & 5 & 36330.81 & & 0.8 & 1.103 & $\mathrm{~N}$ & d \\
\hline${ }^{4} I_{9 / 2} d_{5 / 2}$ & 6 & 36570.10 & 50 & 0.1 & 1.039 & $\mathbf{N}$ & $\mathrm{I} 4 \mathrm{~d} 5$ \\
\hline${ }^{4} \mathrm{~F}_{9 / 2} \mathrm{~d}_{5 / 2}$ & 7 & 36636.87 & 45 & 0.0 & 1.133 & $\mathbf{N}$ & F4d5 \\
\hline $4 f^{11} 5 d$ & 5 & 36655.60 & & 1.5 & 1.006 & $\mathbf{N}$ & d5 \\
\hline${ }^{4} \mathrm{I}_{9 / 2} \mathrm{~d}_{5 / 2}$ & 4 & 37608.12 & 40 & 0.8 & 0.891 & $\mathbf{N}$ & $\mathrm{d}$ \\
\hline $4 f^{11} 5 d$ & 3 & 37812.87 & & 0.2 & 1.188 & $\mathrm{~N}$ & d \\
\hline $4 f^{11} 5 d$ & 2 & 38563.97 & & & & $\mathbf{N}$ & ?d \\
\hline$? 4 f^{11} 5 d$ & 6 & 38781.51 & & 32.1 & 1.191 & $N$ & \\
\hline$? 4 f^{11} 6 \mathrm{~s}$ & 6 & 38853.02 & & 67.0 & 1.179 & $\mathbf{N}$ & \\
\hline $4 f^{11} 5 d$ & 3 & 38924.30 & & 0.1 & 1.187 & $\mathbf{N}$ & d \\
\hline $4 f^{11} 5 d$ & 5 & 39265.81 & & 35.1 & 1.082 & $\mathbf{N}$ & \\
\hline${ }^{4} F_{7 / 2} d_{3 / 2}$ & 4 & 39667.36 & 39 & 9.9 & 1.134 & $\mathbf{N}$ & $\mathrm{F} 3 \mathrm{~d} 3$ \\
\hline${ }^{4} F_{7 / 2} s_{1 / 2}$ & 4 & 40166.45 & 73 & 79.8 & 1.257 & $\mathbf{N}$ & F3s1 \\
\hline $4 \mathrm{f}^{115 \mathrm{~d}}$ & 3 & 40407.72 & & 28.7 & 1.022 & $\mathbf{N}$ & d \\
\hline $4 f^{11} 5 d$ & 4 & 40580.40 & & & & $\mathbf{N}$ & \\
\hline $4 f^{11} 5 d$ & 5 & 40857.10 & & & 1.198 & $\mathbf{N}$ & $d ?$ \\
\hline $4 f^{11} 5 d$ & 5 & 46552.18 & & & & $\mathbf{N}$ & $\mathrm{d} ?$ \\
\hline $4 f^{11} 5 d$ & 4 & 46937.23 & & & & $\mathbf{N}$ & \\
\hline $4 f^{11} 5 d$ & 5 & 48747.15 & & & & $\mathbf{N}$ & $d ?$ \\
\hline $4 f^{11} 7 s$ & & & & & & & \\
\hline${ }^{4} I_{15 / 2} 7 s_{1 / 2}$ & 8 & 102599.84 & & & & $\mathbf{N}$ & I77s \\
\hline${ }^{4} I_{15 / 2} 7 s_{1 / 2}$ & 7 & 102913.92 & & & & $\mathbf{N}$ & $177 \mathrm{~s}$ \\
\hline
\end{tabular}

Notes:

a Level first reported in [2].

b Level first reported in [1].

c Level first reported in [3].

d Level first reported in [2], the $J$-value is revised.

e Level present in the list of classified lines of [2].

$\mathrm{N}$ New energy level. 
Table III. Fitted energy parameters, and their standard errors $\left(\mathrm{cm}^{-1}\right)$ in $\mathrm{Er}$ III. The constrained parameters are indicated by an " $f$ ", for "fixed value" and an " $r$ " for "held in a constant ratio with the parameter above".

\begin{tabular}{|c|c|c|}
\hline Parameter & Value & Standard error \\
\hline \multicolumn{3}{|c|}{ Configuration $4 \mathrm{f}^{12}$} \\
\hline$E_{0}$ & 9458.7 & 10 \\
\hline$E_{1}$ & 6147.1 & $r$ \\
\hline$E_{2}$ & 30.87 & 0.17 \\
\hline$E_{3}$ & 617.4 & 1.7 \\
\hline$\zeta_{4 i}$ & 2245.0 & 4.6 \\
\hline$\alpha$ & 20 & f \\
\hline$\beta$ & $200 f$ & \\
\hline$y$ & -55 & f \\
\hline Number of & & 7 \\
\hline Number of & & 4 \\
\hline r.m.s. deviat & & 22 \\
\hline \multicolumn{3}{|c|}{ Configuration $4 f^{11} 6 p$} \\
\hline$E_{0}$ & 80408.3 & 39.3 \\
\hline$E_{1}$ & 6512.1 & 12.3 \\
\hline$E_{2}$ & 30.18 & \\
\hline$E_{3}$ & 667.2 & 1.2 \\
\hline$F^{2}(\mathrm{f}, \mathrm{p})$ & 5872 & 141 \\
\hline$G^{2}(\mathrm{f}, \mathrm{p})$ & 1811 & 35 \\
\hline$G^{4}(\mathrm{f}, \mathrm{p})$ & 1542 & 91 \\
\hline$\zeta_{4 f}$ & 2394.5 & 3.7 \\
\hline$\zeta_{6 \mathrm{p}}$ & 3672.8 & 6.5 \\
\hline$\alpha f^{11} p$ & 7.5 & 0.7 \\
\hline$\alpha \mathrm{f}^{11}$ & 20 & \\
\hline$\beta \mathrm{f}^{11}$ & 185 & \\
\hline$y \mathrm{f}^{11}$ & -55 & \\
\hline Number of & & 35 \\
\hline Number of & & 9 \\
\hline r.m.s. deviat & & 22 \\
\hline \multicolumn{3}{|c|}{ Configuration $4 f^{11} 5 d+4 f^{11} 6 s$} \\
\hline$E_{0}$ & 38122.0 & 106 \\
\hline$T(6 s-5 d)$ & 2836.1 & 125 \\
\hline$E_{1}$ & 6493.8 & 32 \\
\hline$E_{2}$ & 29.56 & 0.60 \\
\hline$E_{3}$ & 659.0 & 1.1 \\
\hline$F^{2}(\mathrm{f}, \mathrm{d})$ & 20070 & 243 \\
\hline$F^{4}(\mathrm{f}, \mathrm{d})$ & 14602 & 344 \\
\hline$G^{1}(\mathrm{f}, \mathrm{d})$ & 7707 & 194 \\
\hline$G^{3}(\mathrm{f}, \mathrm{d})$ & 8134 & 361 \\
\hline$G^{5}(\mathrm{f}, \mathrm{d})$ & 5633 & 286 \\
\hline$G^{3}(\mathrm{f}, \mathrm{s})$ & 2123 & 95 \\
\hline$\zeta_{4 \mathrm{f}}$ & 2386.0 & 4.4 \\
\hline$\zeta_{s \mathrm{~d}}$ & 1087.3 & 7.9 \\
\hline$\alpha_{\text {TOT }}$ & 6.3 & 0.7 \\
\hline$D^{3}(\mathrm{f}, \mathrm{d})$ & 1626 & $\mathrm{f}$ \\
\hline$X^{2}(\mathrm{f}, \mathrm{d})$ & 2660 & 233 \\
\hline$X^{4}(\mathrm{f}, \mathrm{d})$ & 2360 & 465 \\
\hline$\alpha \mathrm{f}^{11}$ & 20 & f \\
\hline$\beta \mathrm{f}^{11}$ & 185 & $\mathrm{f}$ \\
\hline$\gamma \mathrm{f}^{11}$ & -55 & $f$ \\
\hline$R^{2}(\mathrm{rd}, \mathrm{fs})$ & 0 & $\mathrm{f}$ \\
\hline$R^{3}(\mathrm{fd}, \mathrm{sf})$ & 2878 & 297 \\
\hline \multicolumn{2}{|c|}{ Number of levels in the fit } & 64 \\
\hline \multicolumn{2}{|c|}{ Number of free parameters } & 17 \\
\hline r.m.s deviat & & 35 \\
\hline
\end{tabular}

Table IV. Lines of Er III newly identified in the spectrum of the star HR 465

\begin{tabular}{|c|c|c|c|c|}
\hline$\lambda_{\text {ster }}(\AA)$ & $\begin{array}{l}\text { Intensity } \\
\text { in star }\end{array}$ & $\begin{array}{l}\lambda_{\text {leb }} \\
(\AA)\end{array}$ & Spectrum & $\begin{array}{l}\text { Other } \\
\text { spectrum }\end{array}$ \\
\hline 3262.97 & $0-1$ & 0.95 & Er III & $\mathrm{Tb}$ II \\
\hline 3376.15 & 2 & 0.16 & Er III & Nd \\
\hline 3377.43 & 2 & 0.37 & Er III & $\mathrm{Zr}$ II \\
\hline 3404.85 & $3 d ?$ & 0.90 & Er III & $\mathrm{Zr}$ II \\
\hline 3455.06 & 2 & 0.12 & $\mathrm{Er} I I I+$ & \\
\hline 3460.78 & $1 d ?$ & 0.80 & Er III & Pd I \\
\hline 3473.91 & 4 & {$[0.91]$} & Er III & Ho II, Mn II \\
\hline 3492.75 & $1-2$ & 0.75 & Er III & \\
\hline 3501.14 & $1-$ & 0.16 & Er III & \\
\hline 3639.01 & $5 \mathrm{~d} ?$ & {$[0.98]$} & Er III & See note in Table I \\
\hline 3687.46 & 3 & 0.43 & Er III & $\mathrm{Fe} \mathrm{I}$ \\
\hline 3698.98 & $1-2 ?$ & 0.90 & $\mathrm{Er} \mathrm{II}+$ & \\
\hline 3739.41 & $3-4$ & 0.42 & Er III & \\
\hline 3812.54 & 3 & 0.55 & Er III & \\
\hline 3825.54 & $1-$ & 0.53 & Er III & \\
\hline 3898.40 & 1 & 0.35 & $\mathrm{Er} \mathrm{III+}$ & \\
\hline 3913.48 & 5 & 0.50 & Er III & Ti II \\
\hline 3945.17 & $4-5$ & 0.17 & Er III & \\
\hline 3963.42 & $2 ?$ & 0.46 & Er III+ & \\
\hline 3977.34 & $3 \mathrm{~d} ?$ & 0.31 & Er III & \\
\hline 4065.03 & $3-4 \mathrm{~d}$ to $\mathrm{r}$ & 0.04 & Er III & Ho II \\
\hline 4123.47 & 3 & 0.45 & Er III & \\
\hline 4204.01 & $2 d$ & 3.96 & Er III & $\mathrm{Fe} I, \mathrm{Nd}$ \\
\hline 4356.58 & $3 d ?$ & 0.54 & $\mathrm{Er} \mathrm{III}+$ & \\
\hline 4361.99 & 4 & 2.01 & Er III & Sm II \\
\hline 4443.35 & $4 d ?$ & 0.28 & Er III & Dy III \\
\hline 4463.00 & 5 & 0.01 & Er III & NdII \\
\hline 4493.57 & $3-4$ & 0.61 & Er III + & \\
\hline 4579.81 & 1 & 0.81 & Er III & \\
\hline 4669.11 & $\mathrm{~m}$ & 0.09 & Er III & \\
\hline
\end{tabular}

Configuration $4 f^{11} 5 d+4 f^{11} 6 s$ 